\title{
Multiplexed activation of endogenous genes by CRISPRa elicits potent antitumor immunity
}

\author{
Guangchuan Wang ${ }^{1,2,3,16}$, Ryan D. Chow ${ }^{1,2,3,4,16}$, Zhigang Bai ${ }^{1,2,3,15}$, Lvyun Zhu ${ }^{1,2,3}$, Youssef Errami ${ }^{1,2,3}$, \\ Xiaoyun Dai ${ }^{1,2,3}$, Matthew B. Dong ${ }^{1,2,3,4,5,6}$, Lupeng Ye 101,2,3, Xiaoya Zhang ${ }^{1,2,3}$, Paul A. Renauer ${ }^{1,2,3,7}$, \\ Jonathan J. Park ${ }^{1,2,3,4}$, Li Shen ${ }^{1,2,3}$, Hanghui Ye ${ }^{1,2,3}$, Charles S. Fuchs ${ }^{8,9,10}$ and Sidi Chen $\mathbb{1}^{1,2,3,4,6,7,10,11,12,13,14 \star}$
}

Immunotherapy has transformed cancer treatment. However, current immunotherapy modalities face various limitations. In the present study, we developed multiplexed activation of endogenous genes as an immunotherapy (MAEGI), a new form of immunotherapy that elicits antitumor immunity through multiplexed activation of endogenous genes in tumors. We leveraged CRISPR activation (CRISPRa) to directly augment the in situ expression of endogenous genes, and thereby the presentation of tumor antigens, leading to dramatic antitumor immune responses. Deploying this as a cell-based vaccination strategy showed efficacy in both prophylactic and therapeutic settings. Intratumoral adeno-associated virus delivery of CRISPRa libraries elicited strong antitumor immunity across multiple cancer types. Precision targeting of mutated gene sets eradicated a large fraction of established tumors at both local and distant sites. This treatment modality led to alterations in the tumor microenvironment, marked by enhanced T cell infiltration and antitumor immune signatures. Multiplexed endogenous gene activation is a versatile and highly scalable strategy to elicit potent immune responses against cancer, distinct from all existing cancer therapies.

m mmunotherapy leverages the patient's immune system against cancer, turning certain previously lethal cancer types into manageable diseases ${ }^{1-5}$. Major types of immunotherapy include checkpoint blockade ${ }^{6}$, adoptive cell transfer ${ }^{7}$, human recombinant cytokines and cancer vaccines ${ }^{8}$. These regimens have transformed cancer treatment ${ }^{9-11}$. In particular, checkpoint blockade immunotherapies targeting cytotoxic $\mathrm{T}$ lymphocyte-associated protein 4 (CTLA-4) and programmed cell death protein 1 (PD-1) pathways have yielded clinical benefits across a broad spectrum of cancer types, with durable responses even in late-stage, metastatic and chemoresistant tumors ${ }^{12-15}$. However, only a fraction of patients show sustained clinical responses to the currently available regimens $s^{5}$, urging for new types of immunotherapies.

As a consequence of cumulative genetic and epigenetic aberrations, cancers can be recognized and eliminated by the immune system if mutant or abnormally expressed antigens are adequately presented $^{16,17}$. Recognition of tumor-associated antigens (TAAs) formed by mutations and dysregulated gene expression programs is an essential step for cancer immunotherapy ${ }^{17,18}$. However, the spontaneous immune recognition of tumor antigens is often insufficient to elicit effective immune responses, because the abnormal products may not be adequately presented ${ }^{19}$. Moreover, neoantigen loss often occurs during malignancy ${ }^{18}$. We reasoned that augmenting the expression and thus the presentation of endogenous antigens in tumors could amplify the 'nonself' identity of cancer cells, thereby flagging them for immune destruction ${ }^{20}$.
Neoantigen-targeting approaches based on the delivery of synthetic mutant peptides or transcripts have demonstrated the concept of leveraging personalized neoantigens as cancer treatments ${ }^{21-24}$. However, the efficacy and scalability of these approaches is limited. The CRISPRa system uses a catalytically inactive Cas9 (dCas9) ${ }^{25}$, enabling simple and flexible gene expression regulation through dCas9-transcriptional activators paired with single guide RNAs (sgRNAs) ${ }^{26-29}$. Using CRISPRa, multiplexed augmentation of desired gene sets can be readily achieved by utilizing pools of guide $\mathrm{RNAs}^{27}$. In the present study, we developed CRISPRa-mediated MAEGI, which acts by directly augmenting the expression and presentation of endogenous genes that encode potentially immunogenic antigens. MAEGI showed therapeutic efficacy across three tumor types. Mechanistically, MAEGI treatment elicits antitumor immune responses by recruiting effector $\mathrm{T}$ cells and remodeling the tumor microenvironment.

\section{Results}

CRISPRa enhances in situ antigen presentation and promotes $\mathrm{T}$ cell effector function. To investigate whether CRISPRa can elicit immune responses by enhancing the presentation of TAAs (Fig. 1a), we examined the effect of CRISPRa on the surface presentation of a target antigenic peptide. Triple-negative breast cancer (TNBC) E0771 cells were transduced with CRISPRa lentiviral vectors expressing dCas9-VP64 and MS2-p65-HSF1 (MPH) (E0771-dCas9-VP64-MPH; see Supplementary Fig. 1a). By introducing a model antigen transgene (chicken ovalbumin,

'Department of Genetics, Yale University School of Medicine, New Haven, CT, USA. 2System Biology Institute, Yale University, West Haven, CT, USA. ${ }^{3}$ Center for Cancer Systems Biology, Yale University, West Haven, CT, USA. ${ }^{4}$ MD-PhD Program, Yale University, New Haven, CT, USA. ${ }^{5}$ Department of Immunobiology, Yale University School of Medicine, New Haven, CT, USA. ${ }^{6}$ mmunobiology Program, Yale University, New Haven, CT, USA. ${ }^{7}$ Combined Program in the Biological and Biomedical Sciences, Yale University, New Haven, CT, USA. ${ }^{8}$ Department of Medicine, Yale University School of Medicine, New Haven, CT, USA. ${ }^{9}$ Smilow Cancer Hospital, New Haven, CT, USA. ${ }^{10}$ Yale Comprehensive Cancer Center, Yale University School of Medicine, New Haven, CT, USA. "'Department of Neurosurgery, Yale University School of Medicine, New Haven, CT, USA. ${ }^{12}$ Yale Stem Cell Center, Yale University School of Medicine, New Haven, CT, USA. ${ }^{13}$ Yale Liver Center, Yale University School of Medicine, New Haven, CT, USA. ${ }^{14}$ Yale Center for Biomedical Data Science, Yale University School of Medicine, New Haven, CT, USA. ${ }^{15}$ Present address: Department of General Surgery, Beijing Friendship Hospital of Capital Medical University, Beijing, China. ${ }^{16}$ These authors contributed equally: Guangchuan Wang, Ryan D. Chow. *e-mail: sidi.chen@yale.edu 


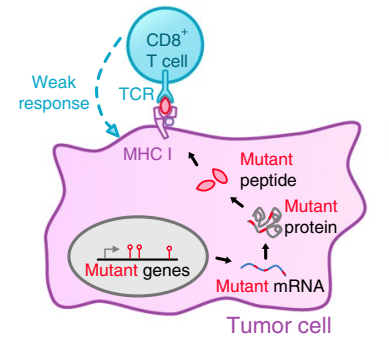

Low expression of TAAs

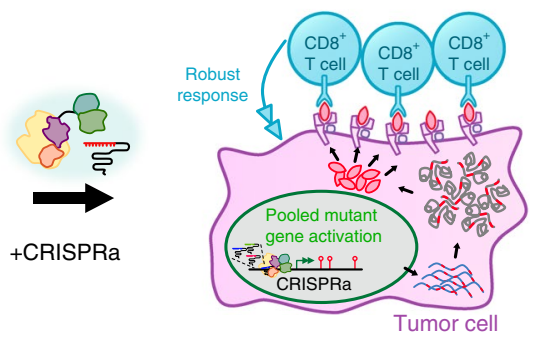

CRISPRa boosts the presentation and immune recognition of TAAs

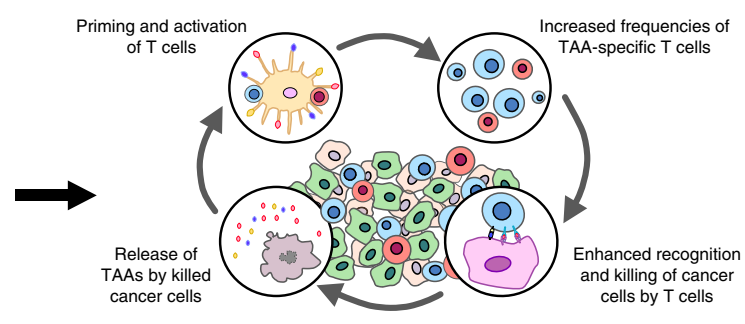

Induction of systemic adaptive immunity against TAAs b

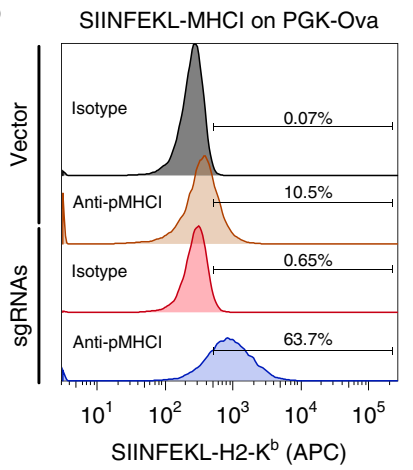

C

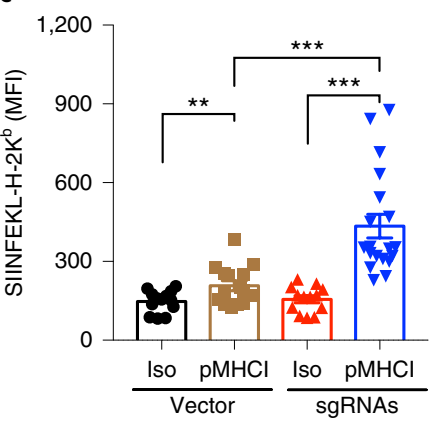

d

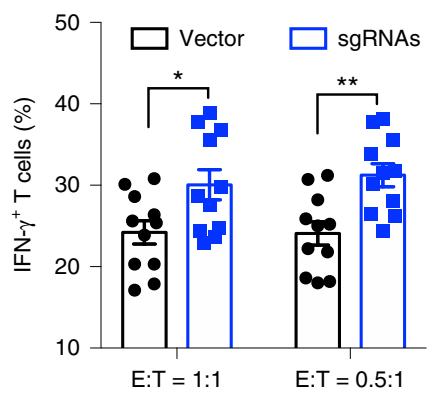

e

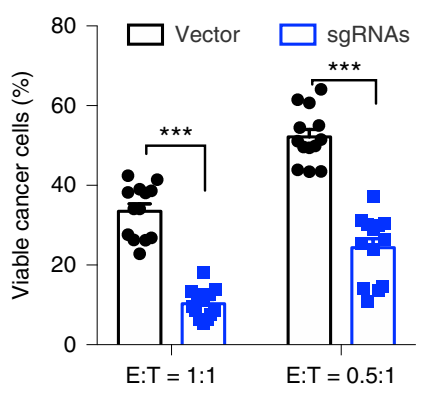

Fig. 1 | CRISPRa augments tumor antigen presentation to promote T cell effector function. a, Schematic of the experimental design for using CRISPRa to enhance the immune recognition of TAAs, eliciting systemic immune responses. $\mathbf{b}, \mathbf{c}$, E0771-dCas9-VP64 cells were transduced with lentivirus to express OVA under a PGK promoter (E0771-OVA), and further transduced with either vector or CRISPRa sgRNAs targeting the PGK promoter. $\mathbf{b}$, Representative flow cytometry analysis of surface staining for OVA-derived SIINFEKL-H-2Kb complex on cells transduced with vector or sgRNAs. c, Mean fluorescence

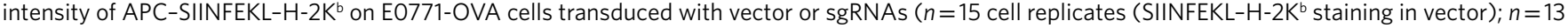
(isotype in vector); $n=19$ (SIINFEKL-H-2Kb staining in CRISPRa sgRNAs); or $n=15$ (isotype in CRISPRa sgRNAs) from four independent experiments; two-sided Mann-Whitney test: SIINFEKL-H-2K ${ }^{b}$ staining versus isotype in vector, $\mathrm{P}=0.0356$; SIINFEKL-H-2Kb staining versus isotype in CRISPRa sgRNAs, $P<0.0001$; SIINFEKL-H-2K $\mathrm{K}^{b}$ staining in CRISPRa sgRNAs versus vector, $\left.P<0.0001\right)$. $\mathbf{d}$, The percentage of IFN- $\gamma$-producing OT-I CD8 ${ }^{+} T$ effector cells after co-culture with the indicated E0771-OVA cancer cells for $3 \mathrm{~h}(n=11$ co-culture samples from three independent experiments with Welch's two-tailed, unpaired $t$-test: CRISPRa sgRNAs versus vector in $E: T=1: 1, P=0.0218 ; C R I S P R a$ sgRNAs versus vector in $E: T=0.5: 1, P=0.002)$. e, The percentage of viable cancer cells (excluding dead cells and apoptotic cells) when co-cultured with OT-I CD8 $8^{+}$T effector cells for $24 \mathrm{~h}$ ( $n=11$ co-culture samples from three independent experiments; Welch's two-tailed, unpaired $t$-test: CRISPRa sgRNAs versus vector in $\mathrm{E}: T=1: 1, P<0.0001$; CRISPRa sgRNAs versus vector in $E: T=0.5: 1, P<0.0001)$. Error bars: all data points in this figure are presented as mean \pm s.e.m. ${ }^{\star} P<0.05,{ }^{\star \star} P<0.01,{ }^{\star \star \star} P<0.001$. Additional supporting data: see Supplementary Fig. 1.

OVA) driven by a phosphoglycerate kinase (PGK) promoter into E0771-dCas9-VP64-MPH cells (E0771-OVA cells), we found that PGK-targeting CRISPRa sgRNAs enhanced the presentation of the target antigenic peptide (SIINFEKL) on the $\mathrm{H}-2 \mathrm{~K}^{\mathrm{b}}$ class I major histocompatibility complex (MHC-I) (Fig. 1b,c and see Supplementary Table 1).

We then used a co-culture assay to investigate the effect of CRISPRa-enhanced antigen presentation on cancer-immune cell interactions. To assess the effector function of T cells, SIINFEKL$\mathrm{H}-2 \mathrm{~K}^{\mathrm{b}}$-specific $\mathrm{CD} 8^{+} \mathrm{T}$ cells were isolated from ovalbumin peptidespecific (OVA) OT-I mice and were co-cultured with E0771-OVA cells for $3 \mathrm{~h}$. E0771-OVA cells expressing PGK-targeting sgRNAs elicited a higher percentage of interferon- $\gamma$ (IFN- $\gamma$ )-producing $\mathrm{T}$ cells (Fig. 1d and see Supplementary Fig. 1b). To assess T cellmediated killing of cancer cells, OT-I T cells were co-cultured with E0771-OVA cells for $24 \mathrm{~h}$ and the viability and apoptosis of the cancer cells analyzed. Consistent with the increase in IFN- $\gamma$ production, CRISPRa treatment led to a lower percentage of viable cancer cells, with most of the cancer cells either killed or undergoing apoptosis (Fig. 1e and see Supplementary Fig. 1c and Supplementary Table 1). These data demonstrate that CRISPRa enhanced the presentation of a target antigen in cancer cells, thereby eliciting a stronger $\mathrm{T}$ cell effector response.
CRISPRa-mediated gene activation leads to immune-mediated tumor clearance. To test the effect of endogenous gene activation on the immunogenicity of cancer cells in vivo, we examined the tumorigenic ability of CRISPRa-transduced cancer cells in immunocompetent C57BL/6J mice (Fig. 2a and see Methods). A mouse, genome-scale lentiviral, CRISPRa sgRNA library (mSAM) ${ }^{27}$ was transduced into E0771-dCas9-VP64-MPH cells (E0771-SAM) (see Supplementary Fig. 1d,e and Supplementary Table 2). After confirming the efficient activation of endogenous genes using this system (Fig. 2b), we transplanted the library-transduced E0771 cells into C57BL/6J mice. In sharp contrast to E0771-vector cells $(0 / 8$ rejection, that is $8 / 8$ engrafted, all with large tumors), the E0771SAM cell pool was rejected in most $(42 / 50,84 \%)$ of the mice, with the remaining mice $(8 / 50,16 \%)$ developing small tumors (Fig. 2c). To examine the contribution of the immune system toward rejection of E0771-SAM cells, E0771-SAM cells were transplanted into immunodeficient Nude (Foxn $1^{n u}$ ) and Rag1 ${ }^{-/-}$mice. Unlike immunocompetent C57BL/6J hosts (5/5 either completely rejected or with small modules), all immunodeficient Nude (Foxn $\left.1^{n u}\right)(4 / 4)$ and Rag1 $^{-/-}$(5/5) mice rapidly grew large tumors from E0771-SAM cells (Fig. 2d). When both $\mathrm{CD}^{+}$and $\mathrm{CD} 8^{+} \mathrm{T}$ cells were depleted in C57BL/6J mice using anti-CD4 and anti-CD8 monoclonal antibodies, all mice receiving E0771-SAM cells formed large tumors 


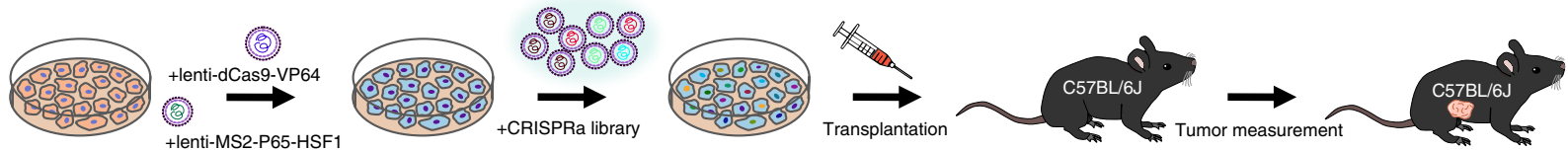

b
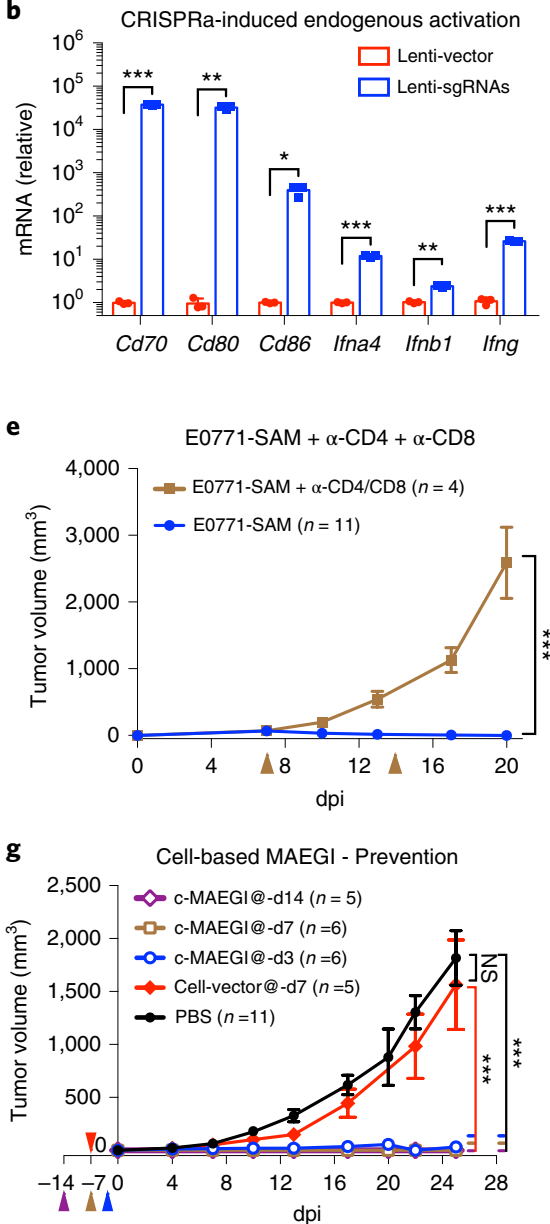

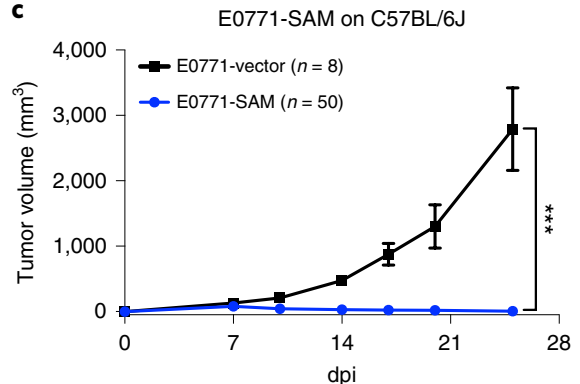

d

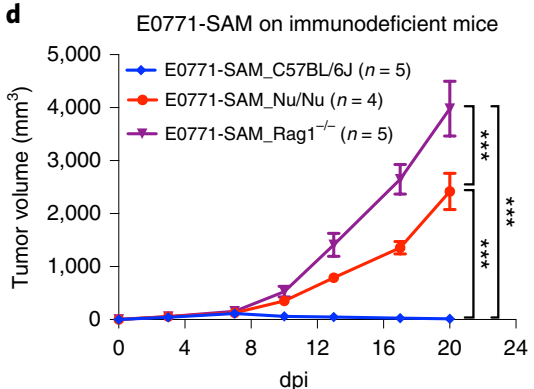

f

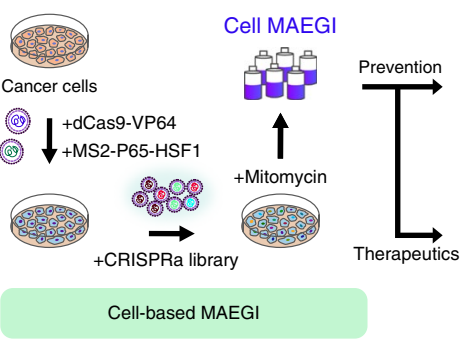

h

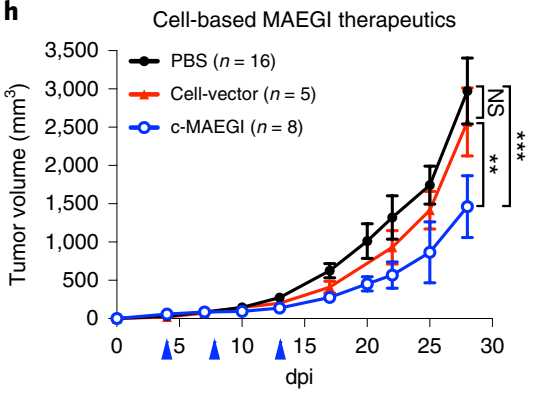

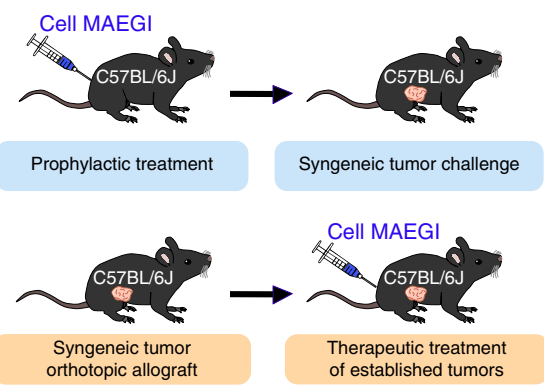

i

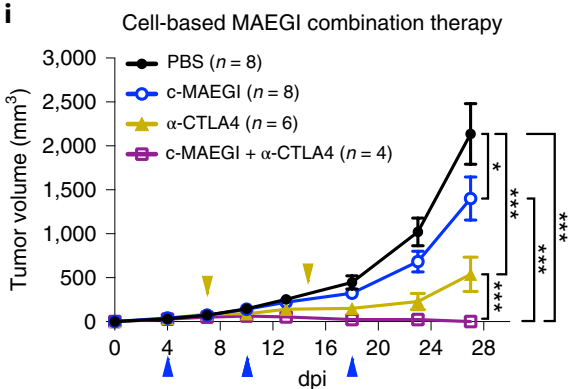

Fig. 2 | MAEGI in a cellular formulation. a, Schematics for generating genome-scale CRISPR activation (SAM), sgRNA library-transduced cells and assessment of tumorigenic capacity. b, Lentiviral CRISPRa-mediated transcriptional activation of $C d 70, C d 80, C d 86$, Ifn $\alpha 4$, Ifn $\beta 1$ and Ifn $\gamma$, normalized to vector-transduced controls ( $n=3$ infection replicates from one representative experiment; two-sided, unpaired, Students $t$-test, sgRNAs versus vector Cd70, $P=0.0006 ; C d 80, P=0.0025 ; C d 86, P=0.026$; Ifn 4 4, $P=0.0007 ;$ Ifn $\beta 1, P=0.003 ;$ Ifn $\gamma, P<0.0001)$. c, Tumor growth curves of vector (E0771-vector, $n=8$ mice) or SAM-transduced E0771-SAM $(n=50)$ transplanted in C57BL/6J mice (two-way ANOVA: $P<0.0001$. d, Tumor growth curves of E0771SAM cells in syngeneic immunocompetent mice (C57BL/6J, $n=5$ mice) and two strains of immunodeficient mice (Nude (Foxn $\left.1^{n u}\right), n=4 ;$ Rag $1^{-1 /}, n=5$; two-way ANOVA: C57BL/6J versus Nude (Foxn $\left.7^{n u}\right), P<0.0001$; C57BL/6J versus Rag1 ${ }^{-1}, P<0.0001$; Nude (Foxn $1^{n u}$ ) versus Rag $1^{-1 /}, P<0.0001$ ). e, Tumor growth curves of E0771-SAM, with $(n=4$ mice) or without $(n=11)$ T cell depletion (two-way ANOVA, untreated versus $\alpha$-CD4 $+\alpha-C D 8$ treated, $P<0.0001)$. f, Schematics of the experimental design for harnessing MAEGI in a cellular vaccine formulation. The E0771 c-MAEGI was generated by treating E0771-SAM cells (E0771 transduced with dCas9-VP64, MS2-P65-HSF1 and a genome-wide CRISPRa library) with mitomycin. The mock cell-vector treatment (Cell-vector) was generated in parallel. $\mathbf{g}$, Growth curves of orthotopic E0771 transplants in mice pre-inoculated with PBS, mock cell-vector vaccine or c-MAEGI at various time points before tumor transplantation (PBS, $n=11$ mice; E0771-vector at dpi=-7 (abbreviated @-d7, $n=5$ ), c-MAEGI@-d14 $(n=5), c-M A E G I @-d 7(n=6)$ and c-MAEGI@-d3 $(n=6)$ (two-way ANOVA: cell-vector@-d7 versus PBS, P=0.2077; c-MAEGI@-d3 versus PBS or vector@-d7, P<0.0001; c-MAEGI@-d7 versus PBS or vector@-d7, P<0.0001; c-MAEGI@-d14 versus PBS or vector@-d7, P<0.0001). h, E0771 tumor-bearing mice were treated with PBS ( $n=16$ mice), cell-vector $(n=5)$ or c-MAEGI $(n=8)$ at indicated times (blue arrows) (two-way ANOVA: PBS versus vector, $P=0.2955$; c-MAEGI versus $P B S, P<0.0001$; c-MAEGI versus vector, $P=0.0005)$. i, Tumor growth curves in E0771 tumorbearing mice that were treated by PBS $(n=8$ mice), c-MAEGI $(n=8), \alpha-C T L A-4(n=6)$ or c-MAEGI $+\alpha-C T L A-4(n=4)$ (blue arrows, c-MAEGI treatment; yellow arrows, $\alpha$-CTLA- 4 treatment; two-way ANOVA: c-MAEGI versus PBS, $P=0.0105 ; \alpha-C T L A-4$ versus PBS, $P<0.0001 ; c-M A E G I+\alpha-C T L A-4$ versus $\alpha$-CTLA-4 alone, $P=0.0005 ; \mathrm{c}-\mathrm{MAEGI}+\alpha-\mathrm{CTLA}-4$ versus c-MAEGI, $P<0.0001)$. Error bars: all data points in this figure are presented as mean \pm s.e.m. ${ }^{\star} P<0.05,{ }^{\star \star} P<0.01,{ }^{\star \star \star} P<0.001$. NS, not significant. dpi, days post injection. Additional supporting data: see Supplementary Fig. 1.

(4/4), in contrast to untreated mice (0/11 with tumors) (Fig. 2e). Together, these results indicate that pooled activation of endogenous genes induced potent tumor rejection in vivo only in immunocompetent hosts.
C-MAEGI has prophylactic and therapeutic efficacy in syngeneic tumor models. Intrigued by the immune rejection of E0771SAM cells, we considered whether CRISPRa-mediated activation of endogenous genes within tumor cells could be harnessed as a 
new approach for immunotherapy (MAEGI). The lentiviral SAM sgRNA library was transduced into E0771-dCas9-VP64-MPH cells to generate E0771-SAM cells, followed by mitomycin treatment to induce senescence, while maintaining the integrity of peptideMHC-I complexes (Fig. 2f), generating c-MAEGI. E0771-vector cells that were treated with mitomycin were generated in parallel as the cell-vector control. Then, c-MAEGI or cell-vector was inoculated into C57BL/6J mice before E0771 tumor induction (Fig. $2 \mathrm{~g}$ and see Methods). In sharp contrast to untreated mice or cell-vector control-treated mice (both $0 \%$ tumor free), c-MAEGI-treated mice had complete protection (100\% tumor free) against subsequent tumor challenges with the unmodified parental E0771 cells (Fig. $2 \mathrm{~g}$ ). This held true across two vaccination regiments, that is 7 or $14 \mathrm{~d}$ before tumor challenge, whereas vaccination $3 \mathrm{~d}$ before tumor challenge granted near-complete protection ( $1 / 6$ mice with a small nodule, 5/6 tumor free) (Fig. $2 \mathrm{~g}$ and see Supplementary Table 1). The results demonstrate that genome-scale activation of endogenous genes using CRISPRa is an effective approach for prophylaxis against tumors with an otherwise identical genetic background.

Given its efficacy as a prophylactic agent, we postulated that c-MAEGI could also potentially be used as a therapeutic intervention against established tumors. Orthotopic E0771 tumors were implanted before treating the mice with c-MAEGI. Using a threedose treatment scheme, tumors in c-MAEGI-treated mice were notably smaller than those in mice treated with cell-vector control or phosphate-buffered saline (PBS) (Fig. 2h and see Supplementary Table 1). To examine the contribution of T cell-mediated immunity in the antitumor response, CD8 ${ }^{+} \mathrm{T}$ cells were depleted in C57BL/6J mice with anti-CD8 antibodies. $\mathrm{CD} 8^{+} \mathrm{T}$ cell depletion abolished the therapeutic efficacy of c-MAEGI (see Supplementary Fig. 1e-h), indicating that $\mathrm{CD}^{+} \mathrm{T}$ cells are essential for c-MAEGI-mediated antitumor responses. We then investigated whether c-MAEGI could be used together with other immunotherapies or immunomodulatory agents. The combination of c-MAEGI + anti-CTLA-4 was more effective than c-MAEGI alone or anti-CTLA-4 alone, leading to complete regression of established tumors (Fig. 2i and see Supplementary Table 1). Collectively, the data demonstrate that c-MAEGI has efficacy in both prophylactic and therapeutic settings.

AAV-mediated, in situ gene activation as an immunotherapeutic modality (AAV-MAEGI). As enhanced antigen presentation by CRISPRa elicited strong immune responses, we reasoned that direct in vivo delivery of CRISPRa components into target tumors could boost the presentation level of TAAs, thereby serving as a therapeutic modality. Adeno-associated viruses (AAVs) are viral vectors capable of mediating efficient transgene delivery ${ }^{30,31}$. To enable direct delivery of MAEGI to tumors, an AAV version of MAEGI was devised by generating an AAV-CRISPRa vector containing the CRISPRa modules (EF1 $\alpha-M P H$ and U6-sgRNABackbone-MS2). The effectiveness of AAV-CRISPRa in activating endogenous genes was confirmed by infecting E0771-dCas9-VP64 cells with CRISPRa AAVs carrying a small pool of sgRNAs (see Supplementary Fig. 2a). In the E0771-OVA model, AAV-delivered sgRNAs targeting the PGK promoter increased the presentation of antigenic peptide (see Supplementary Fig. 2b).

The genome-scale SAM sgRNA library was then cloned into the AAV-CRISPRa vector to produce AAV-g-MAEGI (see Methods). C57BL/6J mice bearing orthotopic E0771-dCas9-VP64 tumors were treated with AAV-g-MAEGI by intratumoral administration (Fig. 3a). AAV-g-MAEGI treatment led to reduced tumor burden compared with either AAV-vector or PBS (Fig. 3b). AAVvector (EF1 $\alpha-\mathrm{MPH}$ and U6-sgRNABackbone-MS2) treatment also showed an antitumor effect, consistent with the baseline immunogenicity of viral vectors carrying the MPH transgenes, but was nevertheless weaker than AAV-g-MAEGI treatment containing the sgRNA library (Fig. 3b). Using ELISpot, it was found that AAV-g-MAEGI elicited higher frequencies of tumor-reactive immune cells (in terms of the frequencies of IFN- $\gamma$-producing splenocytes stimulated by tumor antigens) compared with AAVvector or PBS (Fig. 3c,d). No difference was observed in the frequencies of tumor-specific, IFN- $\gamma$-secreting splenocytes between PBS and AAV-vector (Fig. 3d). These data demonstrate that multiplexed in situ activation of endogenous genes by AAV-g-MAEGI elicits robust and specific antitumor immune responses in vivo.

To evaluate the broader utility of MAEGI, the same treatment modality was tested on other tumor types. On a syngeneic melanoma mouse model (B16F10, expressing dCas9-VP64), AAV-gMAEGI again demonstrated notable efficacy (Fig. 3e). The efficacy of AAV-g-MAEGI was tested further in a syngeneic pancreatic cancer model using the Pan02 cell line. Against established Pan02dCas9-VP64 tumors, it was observed that, although AAV-vector had an antitumor effect compared with PBS, AAV-g-MAEGI has stronger efficacy compared with both AAV-vector and PBS (Fig. $3 f$ and see Supplementary Table 1). Thus, AAV-mediated delivery of genome-scale MAEGI elicits host immune responses against established tumors across multiple aggressive cancer types.

Multiplexed in situ activation of mutated gene sets as proof-ofconcept precision immunotherapy. As individual tumors have unique mutation profiles distinguishing them from normal tissues, we investigated whether MAEGI could be customized to tumorspecific mutated gene sets. This approach was termed precision MAEGI (p-MAEGI) (Fig. 4a). We hypothesized that the precision version could more specifically enhance the presentation of mutant peptides. To this end, whole-exome sequencing of E0771 cells was performed and all genic SNPs, insertions and deletions (indels) called by comparison to healthy mammary fat-pad cells from wildtype C57BL/6J mice sequenced in parallel (Fig. 4a and see Methods), revealing the E0771-specific mutation profile (see Supplementary Fig. 3a,b). The differential mutation data were then harnessed for CRISPRa sgRNA library design, generating a library of 3,839 sgRNAs targeting 1,116 E0771-mutated genes (see Supplementary Table 2). The library was synthesized and pool cloned, successful cloning verified by sequencing and the AAV pool produced (AAVp-MAEGI) (see Supplementary Fig. 3c).

C57BL/6J mice bearing orthotopic E0771 TNBC were treated with AAV-p-MAEGI, along with AAV-vector and PBS controls. Although the AAV-vector itself showed antitumor effects compared with PBS, AAV-p-MAEGI exhibited dramatic efficacy compared with both AAV-vector and PBS (Fig. $4 \mathrm{~b}$ and see Supplementary Fig. 3d). The CR and nCR rates were determined for each treatment (see Methods), which revealed a combined nCR and CR rate of $4 \%$ for PBS, 0\% for AAV-vector and 44\% for AAV-p-MAEGI (Fig. 4c). Three months after the initial tumor transplantation, nine AAV-pMAEGI-treated mice that had undergone CR were re-challenged. All (9/9) mice completely rejected the E0771 tumor re-challenges (Fig. 4d) and retained a long-term complete remission for over $180 \mathrm{~d}$ (Fig. 4d), indicating that AAV-p-MAEGI had induced potent and durable antitumor responses.

The efficacy of AAV-p-MAEGI was abolished in immunodeficient Rag1/- mice (see Supplementary Fig. 3e), indicating that the adaptive immune system is essential for the antitumor effect of AAV-p-MAEGI. Consistent with this, $\mathrm{CD}^{+} \mathrm{T}$ cell infiltration into tumors was observed by histology and immunohistochemistry (see Supplementary Fig. 4a,b). AAV-p-MAEGI-treated tumors also showed reduced proliferation (see Supplementary Fig. 4c,d). Given the importance of adaptive immunity in driving these antitumor responses, we wondered whether intratumoral delivery of AAV-pMAEGI could elicit systemic antitumor immunity and affect distant sites (Fig. 4e). A syngeneic model of E0771 TNBC was used in which mice bear both 'local' and 'distant' breast tumors. After administration of AAV-p-MAEGI only to local tumors, antitumor 
a
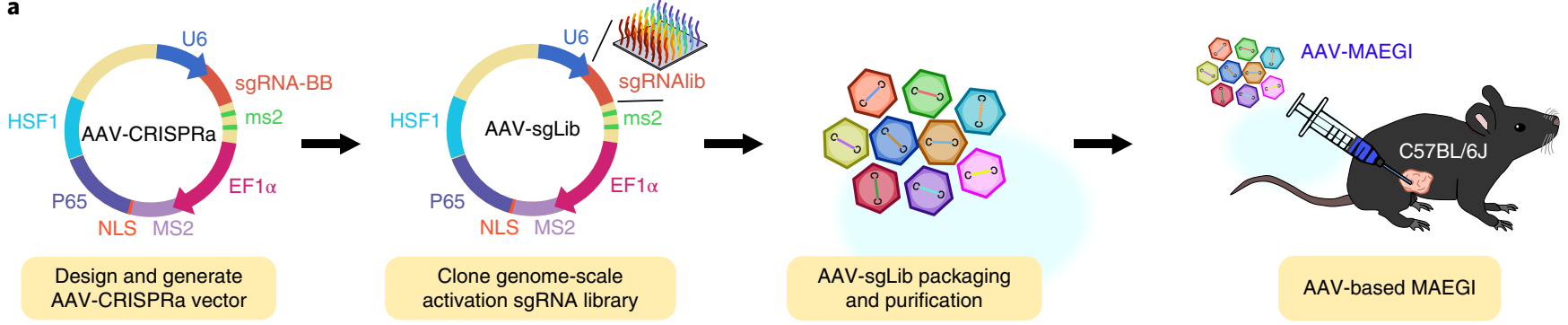

b
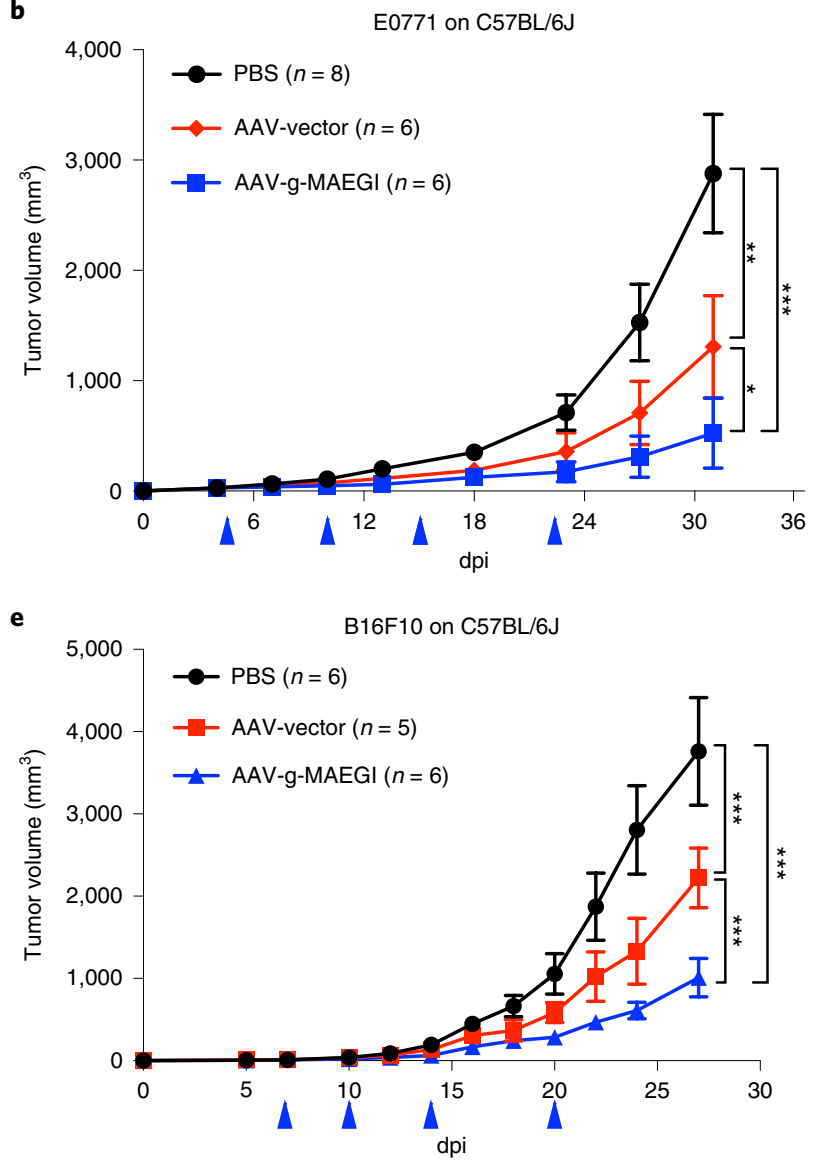

c

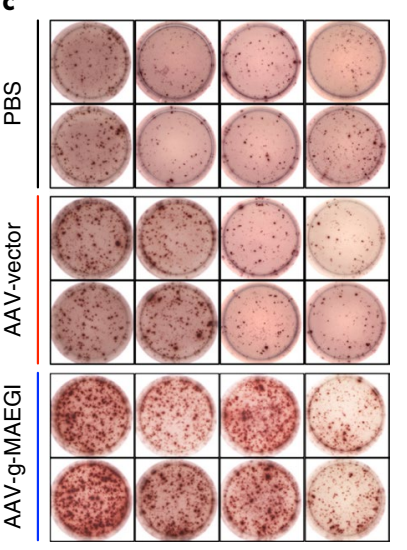

d

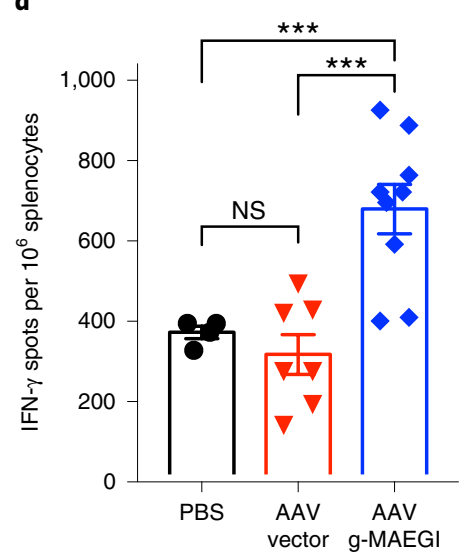

$\mathbf{f}$

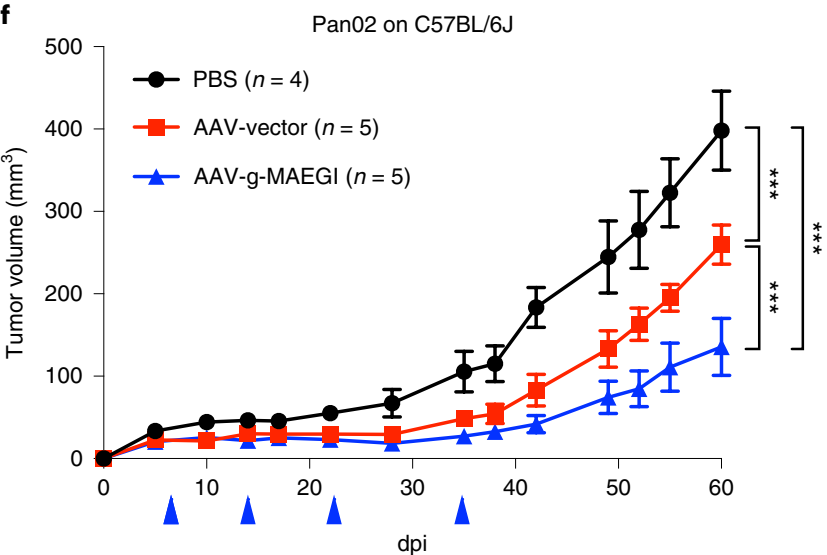

Fig. 3 | Antitumor efficacy of MAEGI in an AAV-based formulation. a, Schematics of the experimental design for intratumoral delivery of MAEGI in an AAV formulation. AAV-g-MAEGI was generated by cloning the genome-scale, activation sgRNA SAM library into an AAV-CRISPRa vector, followed by pooled viral packaging into AAV9. b, TNBC model. Growth curves of orthotopic E0771-dCas9-VP64 tumor transplants in C57BL/6J mice treated with PBS, AAV-vector or AAV-g-MAEGI by intratumoral administration at indicated times (blue arrows) (PBS, $n=8$ mice; AAV-vector, $n=6 ; A A V$-g-MAEGI, $n=6$; two-way ANOVA: PBS versus AAV-vector, $P=0.0013$; AAV-g-MAEGI versus $P B S, P<0.0001$; AAV-g-MAEGI versus $A A V$-vector, $P=0.0303$ ).

$\mathbf{c}, \mathbf{d}$, Tumor-specific immune responses in tumor-bearing $\mathrm{C} 57 \mathrm{BL} / 6 \mathrm{~J}$ mice, assessed by measuring the proportion of IFN- $\gamma$-producing splenocytes with ELISpot assays after stimulation with mitomycin-treated tumor cells. c, Representative images of spots obtained from four mice for each treatment group (PBS, AAV-vector and AAV-g-MAEGI). d, Quantification of ELISpot assays from mice treated with PBS $(n=4$ mice), AAV-vector $(n=7)$ or AAV-g-MAEGI $(n=9)$ (two-sided, unpaired, Student's $t$-test: AAV-vector versus PBS, $P=0.326 ; A A V-g-M A E G I$ versus PBS, $P=0.0009 ; A A V-g-M A E G I$ versus AAVvector, $P=0.0004$; results shown are from the aggregation of two independent experiments). e, Melanoma model. Growth curves of orthotopic B16F10dCas9-VP64 tumor transplants in C57BL/6J mice treated with PBS, AAV-vector or AAV-g-MAEGI at indicated times (blue arrows) (PBS, $n=6$ mice; $A$ AVvector, $n=5$; AAV-g-MAEGI, $n=6$; two-way ANOVA: AAV-vector versus PBS, $P<0.0001$; AAV-g-MAEGI versus PBS, $P<0.0001$; AAV-g-MAEGI versus AAV-vector, $P<0.0001$ ). f, Pancreatic cancer model. Growth curves of Pan02-dCas9-VP64 tumors in C57BL/6J mice treated with PBS ( $n=4$ mice), $\mathrm{AAV}$-vector $(n=5)$ or AAV-g-MAEGI $(n=5)$ at indicated times (blue arrows) (two-way ANOVA: AAV-vector versus PBS, $P<0.0001 ; A A V-g-M A E G I$ versus PBS, $P<0.0001$; AAV-g-MAEGI versus AAV-vector, $P<0.0001)$. Error bars: all data points in this figure are presented as mean \pm s.e.m. ${ }^{\star} P<0.05$, ${ }^{\star \star} P<0.01,{ }^{\star \star \star} P<0.001$. Additional supporting data: see Supplementary Fig. 2.

effects were observed against both local tumors and distant tumors that had not been directly infected by AAV-p-MAEGI (Fig. 4f,g and see Supplementary Table 1). A 67\% CR rate was observed at distant tumors after AAV-p-MAEGI treatment (Fig. 4g). The observation of an abscopal effect indicates that AAV-p-MAEGI had galvanized systemic antitumor immunity.

A critical step in demonstrating the potential of MAEGI as a therapeutic modality is to deliver all of the CRISPRa components 

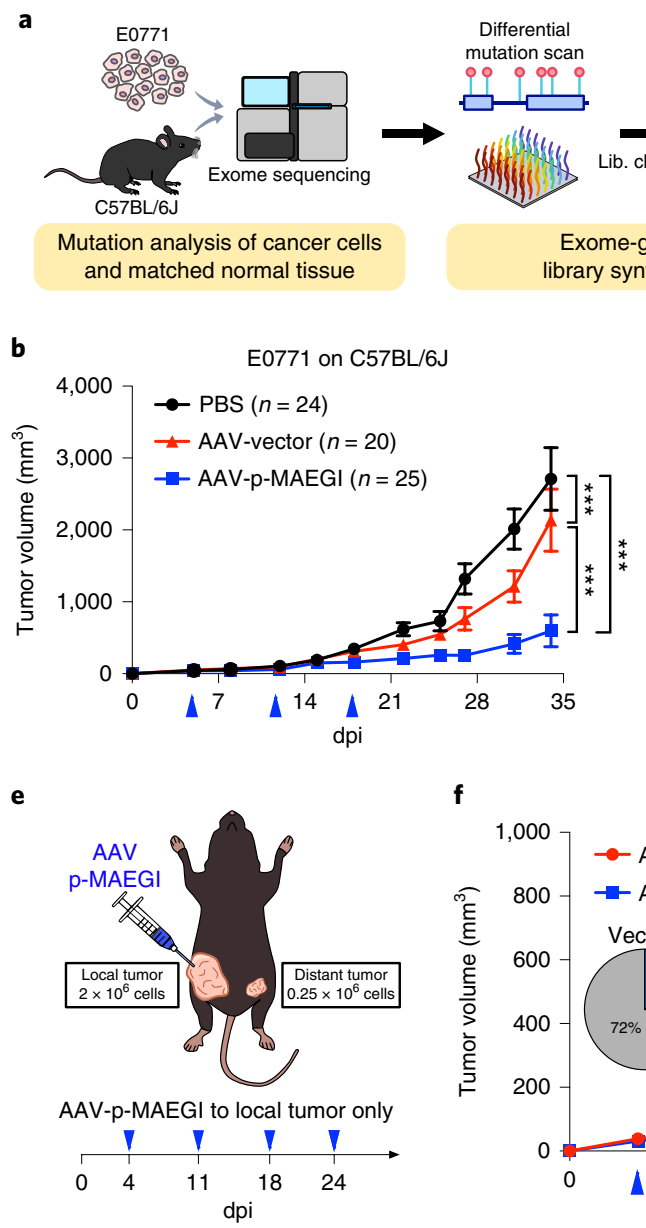

$\mathbf{h}$
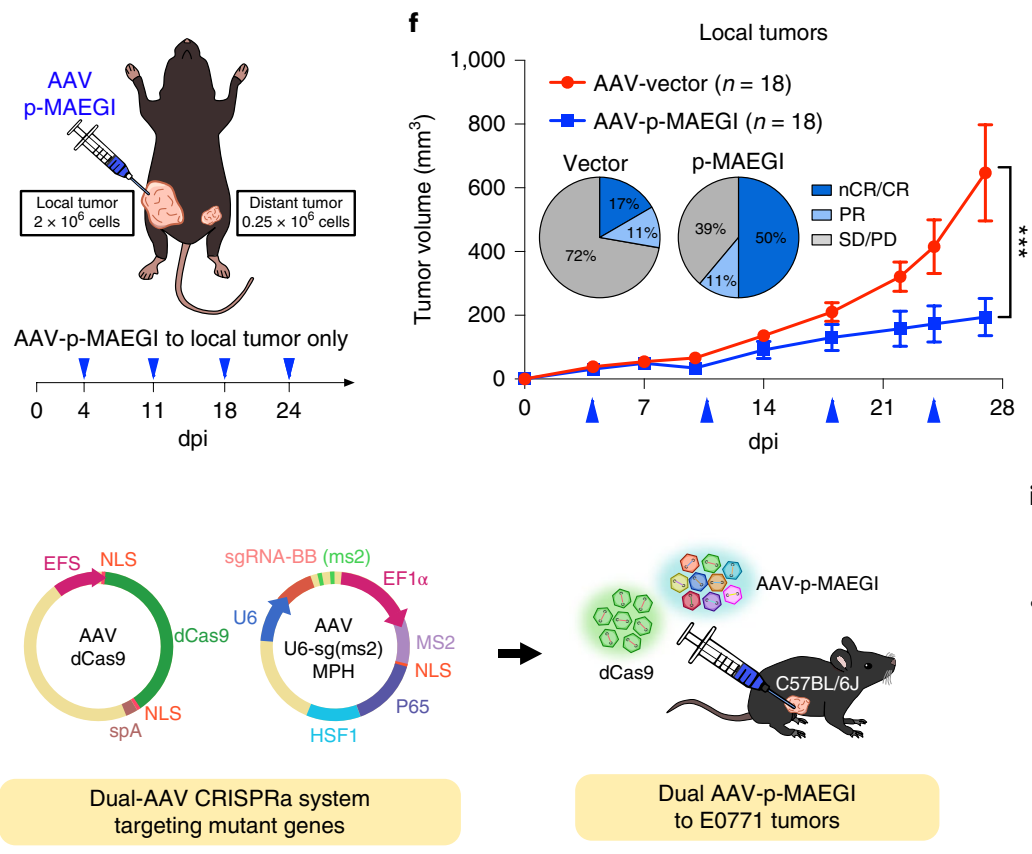
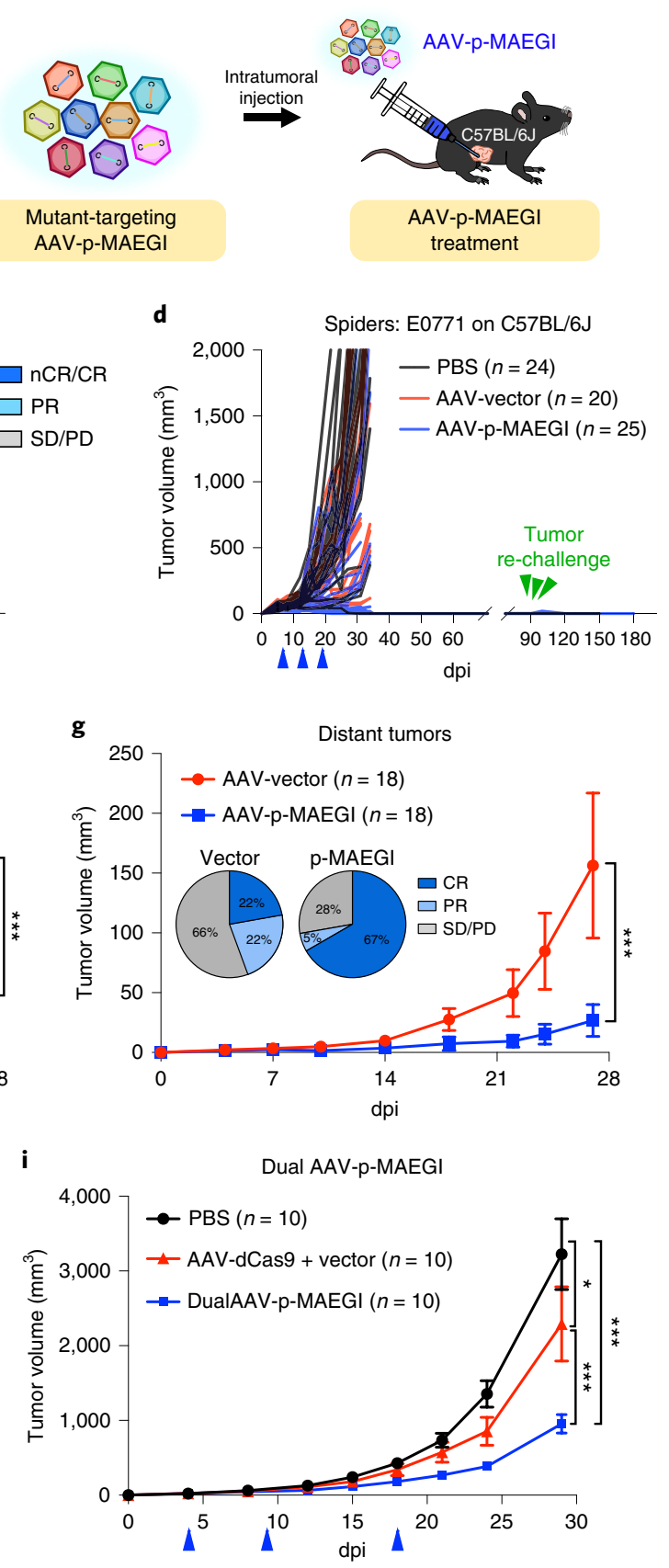

Fig. 4 | Exome-guided p-MAEGI eliminates established tumors. a, Schematics of experimental design. Exome sequencing of cancer cells and normal tissues was performed to identify somatic mutations. A library of CRISPRa sgRNAs targeting the top mutant genes was synthesized and pool cloned into the AAV-CRISPRa vector, and then packaged into AAVs to generate AAV-p-MAEGI. b, Tumor growth curves of E0771-dCas9-VP64 syngeneic tumors in mice treated with PBS $(n=24$ mice), AAV-vector $(n=20)$ or AAV-p-MAEGI $(n=25)$ by intratumoral administration at indicated times (blue arrows) (two-way ANOVA: AAV-vector versus PBS, $P<0.0001$; AAV-p-MAEGI versus PBS, $P<0.0001$; AAV-p-MAEGI versus AAV-vector, $P<0.0001)$. c, Response rates for each treatment, categorized by $\mathrm{nCR} / \mathrm{CR}$, PR or SD/PD. d, Long-term spider growth plots of E0771-dCas9-VP64 tumors treated by PBS, AAV-vector or AAV-p-MAEGI from $\mathbf{b}$. Mice with tumors that had undergone complete response by AAV-p-MAEGI $(n=9)$ were subjected to tumor re-challenges (green arrows). No tumors grew with re-challenge. Treatment days are indicated by blue arrows. $\mathbf{e}$, Schematic of experimental design for evaluating the induction of systemic antitumor immunity by AAV-p-MAEGI. $2 \times 10^{6}$ or $0.25 \times 10^{6}$ E0771-dCas9-VP64 tumor cells were respectively transplanted into the left or right flank of C57BL/6J mice, to model local and distant tumors. AAV-p-MAEGI was administered only into the local tumors at the indicated times (blue arrows). $\mathbf{f}, \mathbf{g}$, Growth curves of E0771-dCas9-VP64 local (f) and distant (g) tumors in mice treated with AAV-vector $(n=18)$ or AAV-p-MAEGI $(n=18)$. Inset: pie charts detailing the response rates for each treatment. f, Two-way ANOVA: AAV-p-MAEGI versus AAV-vector, $P<0.0001$. g, Two-way ANOVA: AAV-p-MAEGI versus AAV-vector, $P=0.0003$. h, Schematics of dual AAV-CRISPRa system for MAEGI delivery into tumor-bearing mice. $\mathbf{i}$, Growth curves of E0771 syngeneic tumors in mice treated by intratumoral injection of PBS $(n=10$ mice), AAV-dCas9 + AAV-vector $(n=10)$ or dual AAV-p-MAEGI $(n=10)$ at the indicated time points (blue arrows) (two-way ANOVA: AAV-dCas9+AAV-vector versus PBS, $P=0.0083$; dual AAV-p-MAEGI versus PBS, $P<0.0001$; dual AAV-pMAEGI versus $A A V$-dCas9 + AAV-vector, $P=0.0002$ ). Error bars: all data points in this figure are presented as mean \pm s.e.m. ${ }^{\star} P<0.05$, ${ }^{\star \star} P<0.01$, ${ }^{\star * \star} P<0.001$. Additional supporting data: see Supplementary Figs. 3-5. 
in vivo. To assess the infection rate of intratumoral AAV delivery, the experiments were repeated using titer-matched AAVs expressing green fluorescent protein (GFP). Four days after intratumoral AAV injection, cancer cells were successfully transduced in all tumors injected, with an infection rate of $4.2 \pm 0.51 \%$ (mean \pm s.e.m.) (see Supplementary Fig. 4e). In contrast, pan-immune $\left(\mathrm{CD} 45^{+}\right)$cells exhibited a lower infection rate of $0.74 \pm 0.25 \%$ (see Supplementary Fig. 4e). At day 12 after two AAV injections, the infection rate increased to $30.4 \pm 5.1 \%$ for tumor cells (see Supplementary Fig. 4f). The off-target infection rate for the spleen, liver, lung and heart in the same cohort of mice was $0 \pm 0 \%, 0.04 \pm 0.04 \%, 0.18 \pm 0.06 \%$ and $0.11 \pm 0.08 \%$, respectively (see Supplementary Fig. $4 \mathrm{~g}$ ). In addition, no severe adverse effects were observed with MAEGI treatment (see Supplementary Table 1).

To enable delivery of all MAEGI components into tumors, a dual AAV system was developed in which one vector expresses dCas9 (AAV-dCas9) and a second vector expresses the sgRNA, as well as MS2-p65-HSF1 (AAV-CRISPRa). To evaluate whether the dual AAV system can effectively activate target transcripts, E0771 cells were co-infected in vitro with both AAV-dCas9 and AAV-CRISPRa, and increased expression of target transcripts was observed 5-7d post-infection (see Supplementary Fig. 5a). Then the therapeutic efficacy of dual AAV-p-MAEGI was examined by co-injection of AAV-dCas9 and AAV-p-MAEGI into unmodified E0771 tumors (Fig. 4h). Similar to the results obtained using tumor cells with lentivirally transduced dCas9-VP64, the co-injection of AAV-dCas9 and AAV-p-MAEGI showed potent antitumor efficacy compared with both AAV-dCas9+AAV-vector and PBS, whereas the co-injection of AAV-dCas9+ AAV-vector showed moderate antitumor effects compared with PBS (Fig. 4i and see Supplementary Table 1). Taken together, these data demonstrate that, by using a dual AAV system, all components of MAEGI can be efficiently delivered into tumors in vivo, resulting in antitumor responses.

AAV-p-MAEGI remodels the tumor-immune microenvironment. To examine the adaptive immune responses over a time course, flow cytometry analysis was performed of tumor-infiltrating $\mathrm{T}$ cell populations (see Supplementary Fig. 5b). AAV-p-MAEGI treatment augmented $\mathrm{CD}^{+}$and $\mathrm{CD}^{+} \mathrm{T}$ cell infiltration in tumors from day 29 onwards, compared with AAV-vector or PBS treatment (Fig. 5a,b). Other immune cell populations were also examined at day 19, because this is the time point with initial observation of tumor regression (see Supplementary Fig. 5c). AAV-p-MAEGI

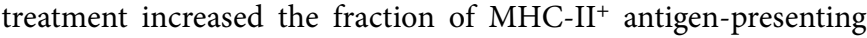
cells (APCs) over AAV-vector treatment (Fig. 5c), with a trend of increased dendritic cells (see Supplementary Fig. 5d), but no significant changes in macrophages, monocytes or neutrophils (see Supplementary Fig. 5e-g and Supplementary Table 1). These results indicate that AAV-p-MAEGI treatment increased T cell infiltration into tumors and altered the composition of tumor-infiltrating immune cells (TIICs), ultimately resulting in tumor regression.

Given these findings, we considered whether the adaptive immune responses induced by AAV-p-MAEGI were tumor specific. To test this, ELISpot assays were performed on both splenocytes and FACS-isolated TIICs, using either E0771 cancer cells or matched normal tissues (primary C57BL/6J wild-type cells isolated from mammary fat pads) as the antigen source (Fig. $5 \mathrm{~d}$ ). In both AAV-vector and AAV-p-MAEGI conditions, the ELISpot assays showed more IFN- $\gamma$-producing splenocytes and TIICs after stimulation by E0771 tumor cells, compared with stimulation with normal primary cells (Fig. 5e-h). Importantly, when stimulated with E0771 cancer cells, AAV-p-MAEGI-treated mice had higher frequencies of IFN- $\gamma$-secreting immune cells than AAV-vector treated mice. This effect was particularly pronounced in TIICs, where AAV-p-MAEGI augmented the frequencies of IFN- $\gamma$ secreting immune cells in response to E0771 stimulation, but not to matched normal cells (Fig. 5g,h). The antitumor specificity of AAV-p-MAEGI was similarly observed in splenocytes (Fig. 5e,f and see Supplementary Table 1). The ELISpot data therefore suggest that tumor-antigen specific immune responses were elicited by AAV-p-MAEGI.

To understand the repertoire of $\mathrm{T}$ cells recruited by MAEGI, $\mathrm{T}$ cell antigen receptor sequencing (TCR-seq) was performed in mice treated with AAV-p-MAEGI, AAV-g-MAEGI, AAV-vector or PBS (Fig. 6a and see Methods). TCR clonal proportions were assessed and various metrics calculated (Fig. 6b-d). In comparison to PBS or AAV-vector treatment, AAV-p-MAEGI increased TCR diversity in both spleens and tumor-infiltrating lymphocytes (TILs) as measured by the Chaol index ${ }^{32}$ (Fig. $6 \mathrm{c}$ and see Supplementary Table 2). In comparison to AAV-g-MAEGI-treated mice (genomewide AAV-MAEGI), splenocytes from AAV-p-MAEGI-treated mice (precision AAV-MAEGI) had higher Chaol indices, with no notable difference among TILs (Fig. 6c). Next, the Gini-Simpson indices were calculated, quantifying the evenness of TCR clonal abundances $^{32}$. TILs from AAV-p-MAEGI-treated mice had higher Gini-Simpson indices compared with PBS- or AAV-vectortreated mice, but not compared with AAV-g-MAEGI-treated mice (Fig. 6d), indicating that MAEGI is associated with the recruitment of diverse T cell populations. Intrigued by the increased TCR diversity and clonal evenness among TILs from AAV-p-MAEGI-treated mice, we considered whether these changes were due to differences in T cell infiltration, in accordance with the FACS data. We examined the number of unique CDR3 clonotypes identified in each sample (that is TCR richness) (Supplementary Fig. 6) ${ }^{32}$, finding that AAV-p-MAEGI mice had more unique clonotypes than PBS or AAV-vector samples in both spleen and TIL samples (Fig. 6e,f). Collectively, the TCR-seq data affirmed the conclusion from the FACS analysis that AAV-p-MAEGI enhanced T cell infiltration into tumors, while additionally revealing the richness and diversity of these tumor-infiltrating T cells.

To further investigate the effect of AAV-p-MAEGI on the tumor microenvironment, single cell RNA sequencing (scRNA-seq) was performed to simultaneously profile the composition and transcriptome of TIICs (Fig. 7a and see Methods). Total TIICs were isolated by FACS of CD $45^{+}$cells and scRNA-seq was performed, recovering a total of 4,381 cells from AAV-p-MAEGI- and 3,482 cells from AAV-vector-treated mice ( $n=3$ mice pooled for each condition; see Supplementary Table 2). Imputation and k-means clustering were performed to identify a total of nine clusters. Analysis of differentially expressed genes in each cluster revealed the cellular identities of each cluster (Fig. $7 \mathrm{~b}$ and see Supplementary Fig. 7a-f). After excluding a population of cells ( $\mathrm{k}$-means cluster 7 , or $\mathrm{k} 7$ ) negative for CD45 gene expression (encoded by Ptprc) (see Supplementary Fig. $7 \mathrm{~b}$ ), the final dataset comprised the transcriptomes of 4,065 TIICs from AAV-p-MAEGI- and 2,799 TIICs from AAV-vectortreated mice (Fig. 7c).

Given that the FACS and TCR-seq analyses had revealed increased T cell tumor infiltration with AAV-p-MAEGI compared with AAV-vector, we sought to investigate whether these findings were recapitulated by scRNA-seq. To perform in silico analysis on $\mathrm{T}$ cell populations, messenger RNA transcript expression levels were used to filter cells ('mRNA gate') in $\mathrm{k} 6$ or $\mathrm{k} 9$, because these clusters comprise the cells that robustly express $C d 3 e, C d 4$ and Cd8a mRNA (Fig. $7 \mathrm{~d}$ ). On examination of the $C d 3 e^{+}$cells within $\mathrm{k} 6$ and $\mathrm{k} 9$, these cells were then mRNA gated into $C d 8 a^{+} C d 4^{-}$and $C d 4^{+} C d 8 a^{-}$populations. Visualization of these cell groups revealed clear separation of putative $\mathrm{CD}^{+} \mathrm{T}$ cells (defined by $\mathrm{Cd} 8 \mathrm{a}^{+} \mathrm{Cd} 3 e$ ${ }^{+} C d 4^{-}$mRNA gating) from $\mathrm{CD} 4^{+} \mathrm{T}$ cells (defined by $\mathrm{Cd} 4^{+} \mathrm{C} d 3 e^{+} \mathrm{C}$ $d 8 a^{-}$mRNA gating) (Fig. $7 \mathrm{~d}$ ). The relative abundance of these different $\mathrm{T}$ cell populations in $\mathrm{k} 6$ and $\mathrm{k} 9$ was quantified, which showed that AAV-p-MAEGI mice had notably more $C d 3 e^{+} \mathrm{T}$ cells, putative $\mathrm{CD}^{+} \mathrm{T}$ cells and putative $\mathrm{CD} 4^{+} \mathrm{T}$ cells (Fig. 7e). Furthermore, 


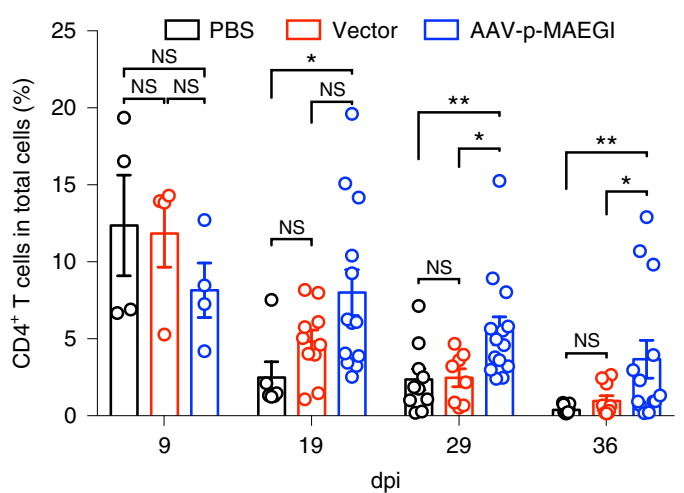

b

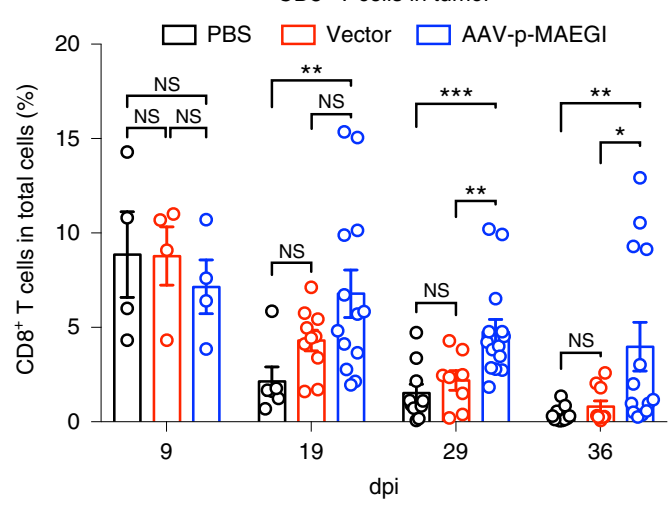

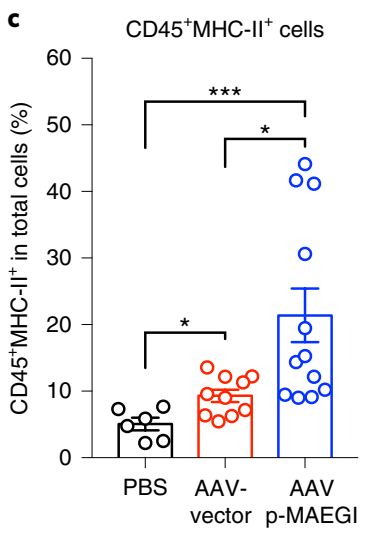

d

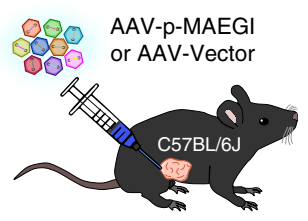

Treat tumor-bearing mice
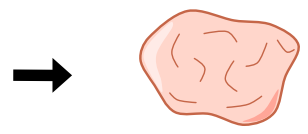

Collect tumors and spleens

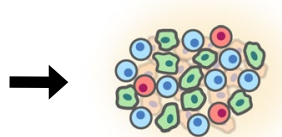

Extract splenocytes and TIICs

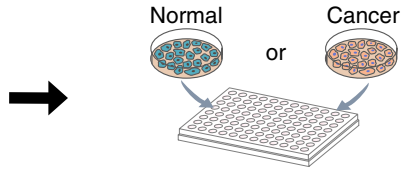

Stimulate with normal primary pot e

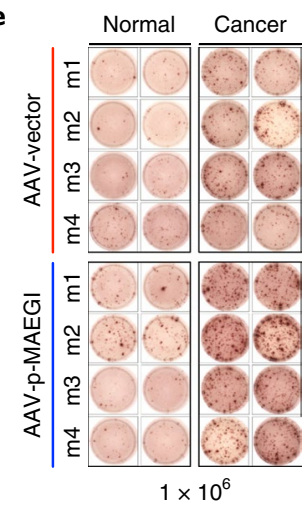

splenocytes per replicate f

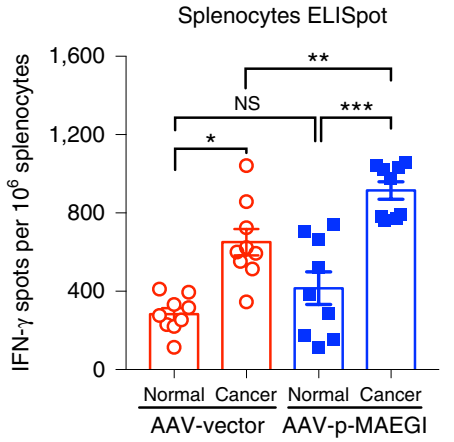

g

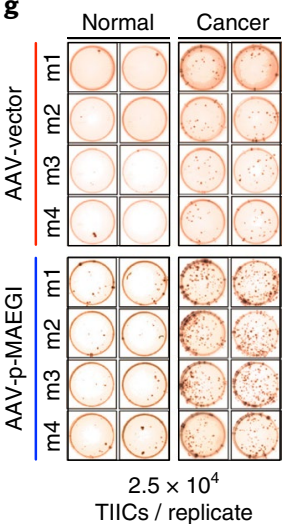

h

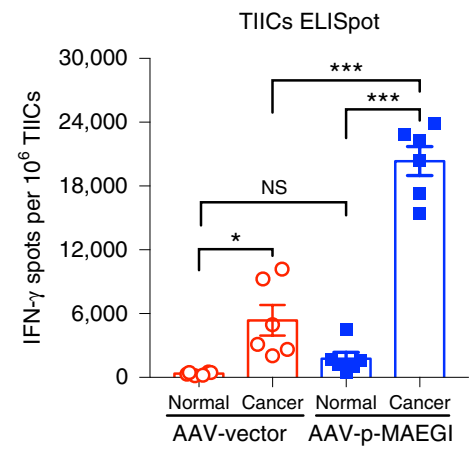

Fig. 5 | AAV-p-MAEGI treatment promotes infiltration of tumor-reactive immune populations. a, Flow cytometry time-course quantification of CD4 ${ }^{+}$ T cells out of total tumor cells from mice treated with PBS, AAV-vector or AAV-p-MAEGI (two-tailed Mann-Whitney test: AAV-p-MAEGI ( $n=4$ mice) versus PBS $(n=4)$ at dpi $9(P=0.6857)$, AAV-p-MAEGI $(n=13)$ versus PBS $(n=6)$ at dpi $19(P=0.0047)$, AAV-p-MAEGI $(n=14)$ versus PBS $(n=10)$ at dpi $29(P=0.0048)$, AAV-p-MAEGI $(n=13)$ versus PBS $(n=11)$ at dpi $36(P=0.0015)$; AAV-p-MAEGI $(n=4)$ versus AAV-vector $(n=4)$ at $d p i 9$ $(P=0.2000)$, AAV-p-MAEGI $(n=13)$ versus AAV-vector $(n=10)$ at dpi $19(P=0.2569)$, AAV-p-MAEGI $(n=14)$ versus AAV-vector $(n=8)$ at dpi 29 $(P=0.0159), A A V-p-M A E G I(n=12)$ versus AAV-vector $(n=10)$ at dpi $36(P=0.0422))$. b. Flow cytometry time course quantification of $C D 8^{+}$T cells out of total tumor cells from mice treated with PBS, AAV-vector or AAV-p-MAEGI (two-tailed Mann-Whitney test: AAV-p-MAEGI ( $n=4$ mice) versus PBS $(n=4)$ at dpi $9(P=0.6857)$; AAV-p-MAEGI $(n=13)$ versus PBS $(n=6)$ at dpi $19(P=0.0047)$; AAV-p-MAEGI $(n=14)$ versus PBS $(n=10)$ at $d p i 29$ $(P=0.0005)$; AAV-p-MAEGI $(n=13)$ versus PBS $(n=11)$ at dpi $36(P=0.0014)$; AAV-p-MAEGI $(n=4)$ versus AAV-vector $(n=4)$ at dpi $9(P=0.4857)$; AAV-p-MAEGI $(n=13)$ versus AAV-vector $(n=10)$ at dpi $19(P=0.2569) ; A A V-p-M A E G I(n=13)$ versus PBS $(n=11)$ at dpi $29(P=0.0064) ;$ AAV-pMAEGI $(n=12)$ versus AAV-vector $(n=10)$ at dpi $36(P=0.0200)$ ). c, Percentage of CD45+MHC-II+ APCs in tumors from mice treated with PBS $(n=6$ mice), AAV-vector $(n=10)$ or AAV-p-MAEGI $(n=12)$ (two-tailed Mann-Whitney test: AAV-vector versus PBS, $P=0.0225 ; A A V-p-M A E G I$ versus PBS, $P=0.0001$; AAV-p-MAEGI versus AAV-vector, $P=0.0147$ ). $\mathbf{d}$, Schematic of experimental design using ELISpot to assess antitumor specificity of the immune response elicited by AAV-p-MAEGI or AAV-vector. e,g Representative images of IFN- $\gamma$ ELISpots on splenocytes (e) or TIICs (g) from AAV-vectoror AAV-p-MAEGI-treated mice bearing E0771-dCas9-VP64 tumors, stimulated with either normal primary cells from wild-type C57BL/6J fat pad tissue (normal) or E0771 cells (cancer). f, Quantification of IFN- $\gamma$ ELISpots on splenocytes from AAV-vector- $(n=9$ mice) or AAV-p-MAEGI $(n=9)$-treated mice bearing E0771-dCas9-VP64 tumors, stimulated with primary C57BL/6J normal cells or cancer cells (two-sided, unpaired, Student's t-test: AAV-p-MAEGI versus $A A V$-vector stimulated by normal cells, $P=0.1667$; AAV-p-MAEGI versus $A A V$-vector stimulated by E0771 cancer cells, $P=0.0057$; cancer versus normal cells stimulation within the AAV-vector group, $P=0.0004$; cancer versus normal cell stimulation within the AAV-p-MAEGI group, $P=0.0002$; results shown are aggregated from two independent experiments). $\mathbf{h}$, Quantification of IFN- $\gamma$ ELISpots for TIICs from AAV-vector- ( $n=6$ ) or AAV-pMAEGI $(n=6)$-treated mice bearing E0771-dCas9-VP64 tumors that were stimulated with primary C57BL/6J normal cells or cancer cells (two-sided, unpaired, Student's $t$-test: AAV-p-MAEGI versus AAV-vector stimulated by normal cells, $P=0.0577 ; A A V-p-M A E G I$ versus AAV-vector stimulated by cancer cells, $P<0.0001$; cancer versus normal cell stimulation for the AAV-vector group, $P=0.0175$; cancer versus normal cell stimulation for the AAV-pMAEGI group, $P<0.0001$ ). Error bars: all data points in this figure are presented as mean \pm s.e.m. ${ }^{\star} P<0.05$, ${ }^{\star \star} P<0.01$, ${ }^{\star \star \star} P<0.001$. Additional supporting data: see Supplementary Fig. 5. 

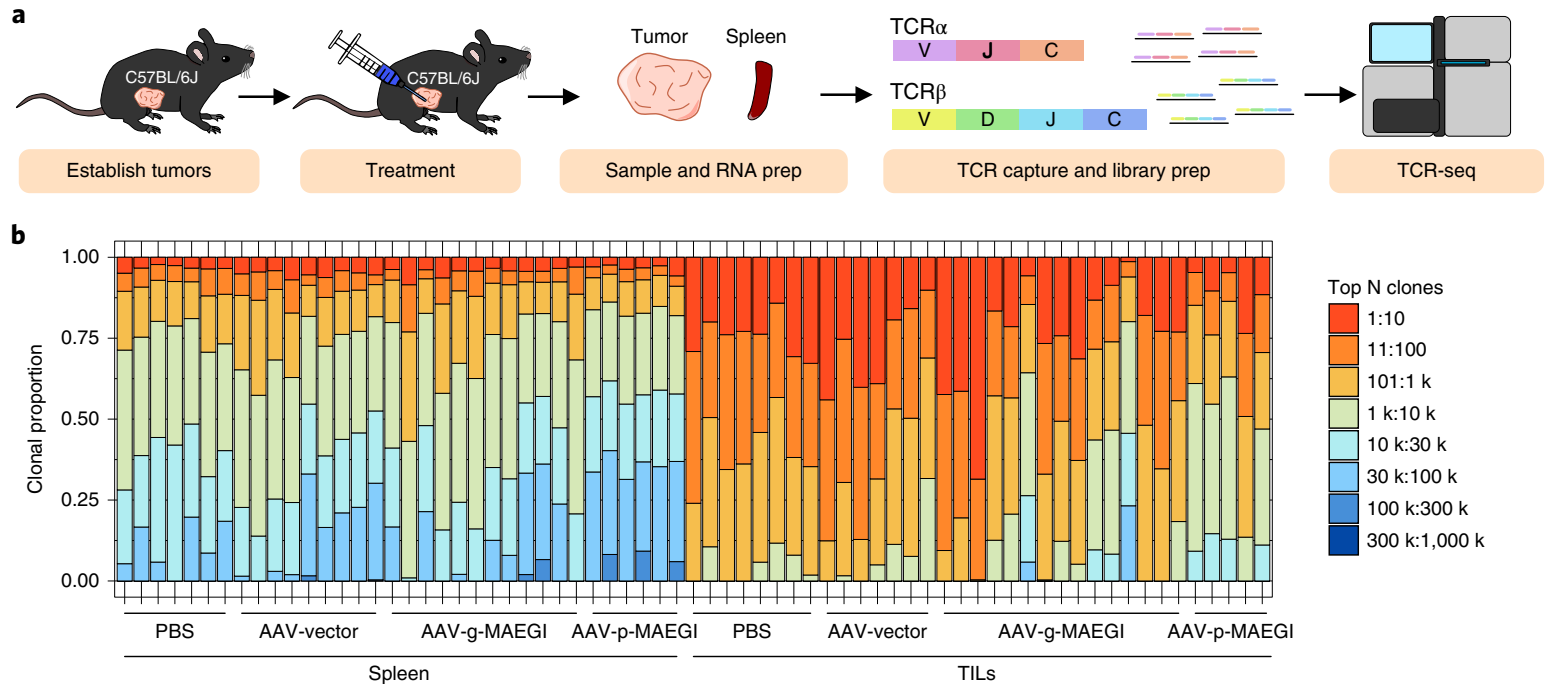

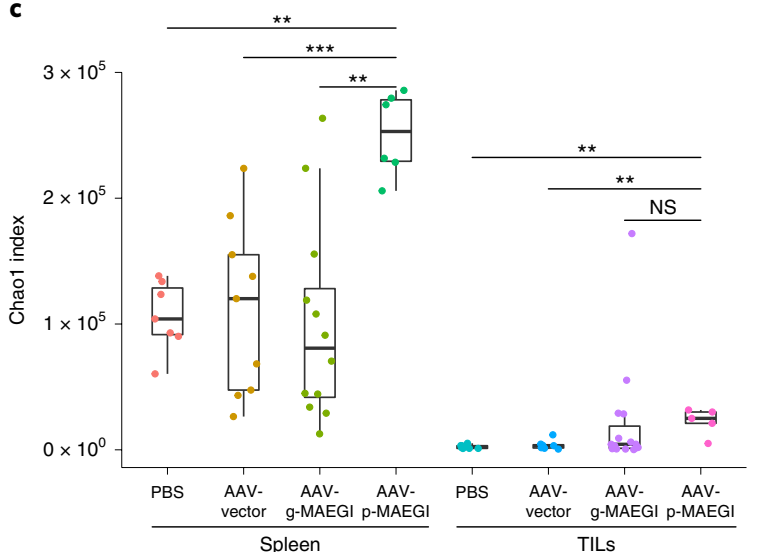

e

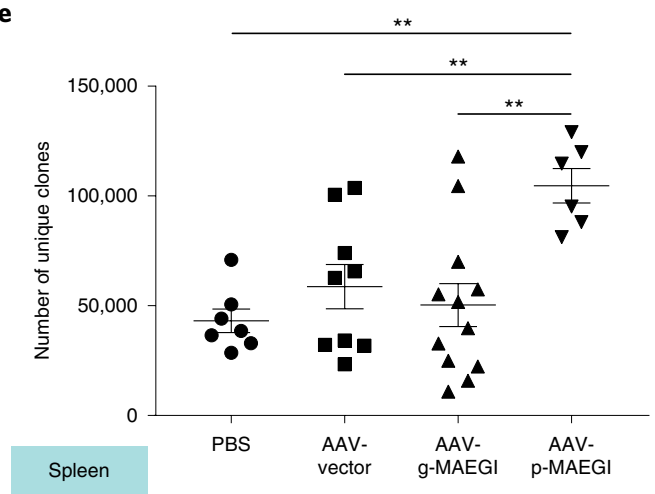

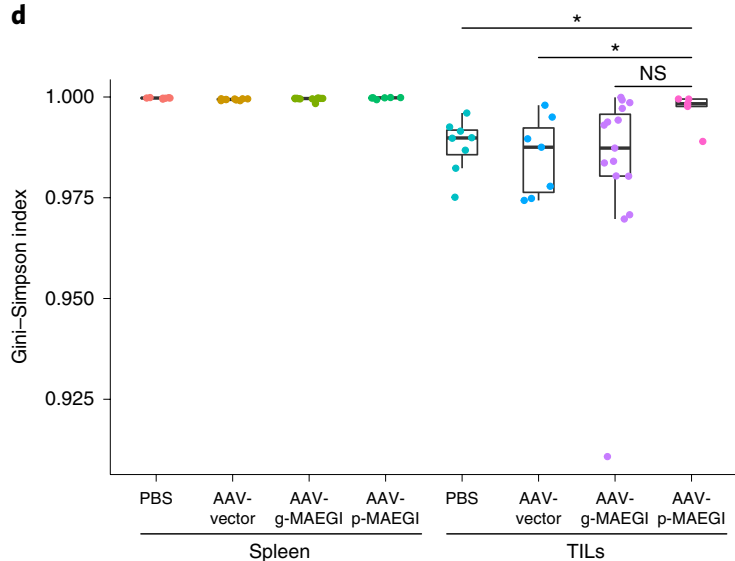

f

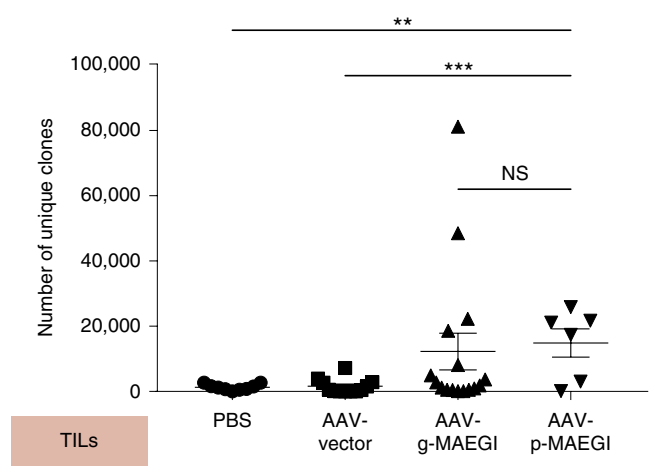

Fig. 6 | Interrogation of the T cell repertoire in MAEGI-treated mice by TCR-seq. a, Schematics of TCR-seq experimental design. Mice bearing E0771dCas9-VP64 tumors were treated with PBS, AAV-vector, AAV-g-MAEGI (genome wide) or AAV-p-MAEGI (exome guided). Spleens and tumors were harvested and subjected to RT-based TCR $\alpha / T C R \beta$ capture followed by Illumina sequencing. In spleen samples, $n=7$ (PBS-treated mice), $n=9$ (AAVvector-treated mice), $n=12$ (AAV-g-MAEGI-treated mice), $n=6$ (AAV-p-MAEGI-treated mice) from two independent experiments. In the TIL samples ( $n=7$ (PBS-treated mice), $n=7$ (AAV-vector-treated mice), $n=15$ (AAV-g-MAEGI-treated mice), $n=5$ (AAV-p-MAEGI-treated mice)) from two independent experiments. b, Global clonal proportion plot showing the relative frequencies of the top $N$ clones. c, Boxplot of Chao1 indices (TCR diversity) for each group (unpaired, two-tailed, Mann-Whitney test, spleens: AAV-p-MAEGI versus PBS, $P=0.0012 ; A A V-p-M A E G I$ versus AAV-vector, $P=0.0008$; AAV-p-MAEGI versus AAV-g-MAEGI, $P=0.0013$; unpaired, two-tailed, Mann-Whitney test, TILs: AAV-p-MAEGI versus PBS, $P=0.0031 ; A A V-p-M A E G I$ versus AAV-vector, $P=0.0051$; AAV-p-MAEGI versus AAV-g-MAEGI, $P=0.0983$ ). d, Boxplot of Gini-Simpson indices (TCR evenness) for each group (two-tailed, Mann-Whitney test, TILs: AAV-p-MAEGI versus PBS, $P=0.0295 ;$ AAV-p-MAEGI versus AAV-vector, $P=0.0303$; AAV-p-MAEGI versus AAVg-MAEGI, $P=0.0526$ ). e,f, Dot plots of the number of unique clonotypes identified (TCR richness) in each spleen sample (e) or TIL sample (f), compared across treatment conditions. Statistical significance was assessed using the unpaired, two-tailed, Student's t-test: spleen: AAV-p-MAEGI versus PBS, $P=0.002 ; A A V-p-M A E G I$ versus AAV-vector, $P=0.006$ AAV-p-MAEGI versus AAV-g-MAEGI, $P=0.0023 ;$ TILs: AAV-p-MAEGI versus PBS, $P=0.002$; AAV-p-MAEGI versus AAV-vector, $P=0.0006$; AAV-p-MAEGI versus AAV-g-MAEGI, $P=0.7875$. All boxplots are Tukey boxplots (interquartile range (IQR) boxes with $1.5 \times I Q R$ whiskers). Error bars: data points in this figure are presented as mean \pm s.e.m. ${ }^{\star} P<0.05,{ }^{\star \star} P<0.01,{ }^{\star \star \star} P<0.001$. Additional supporting data: see Supplementary Fig. 6. 
a
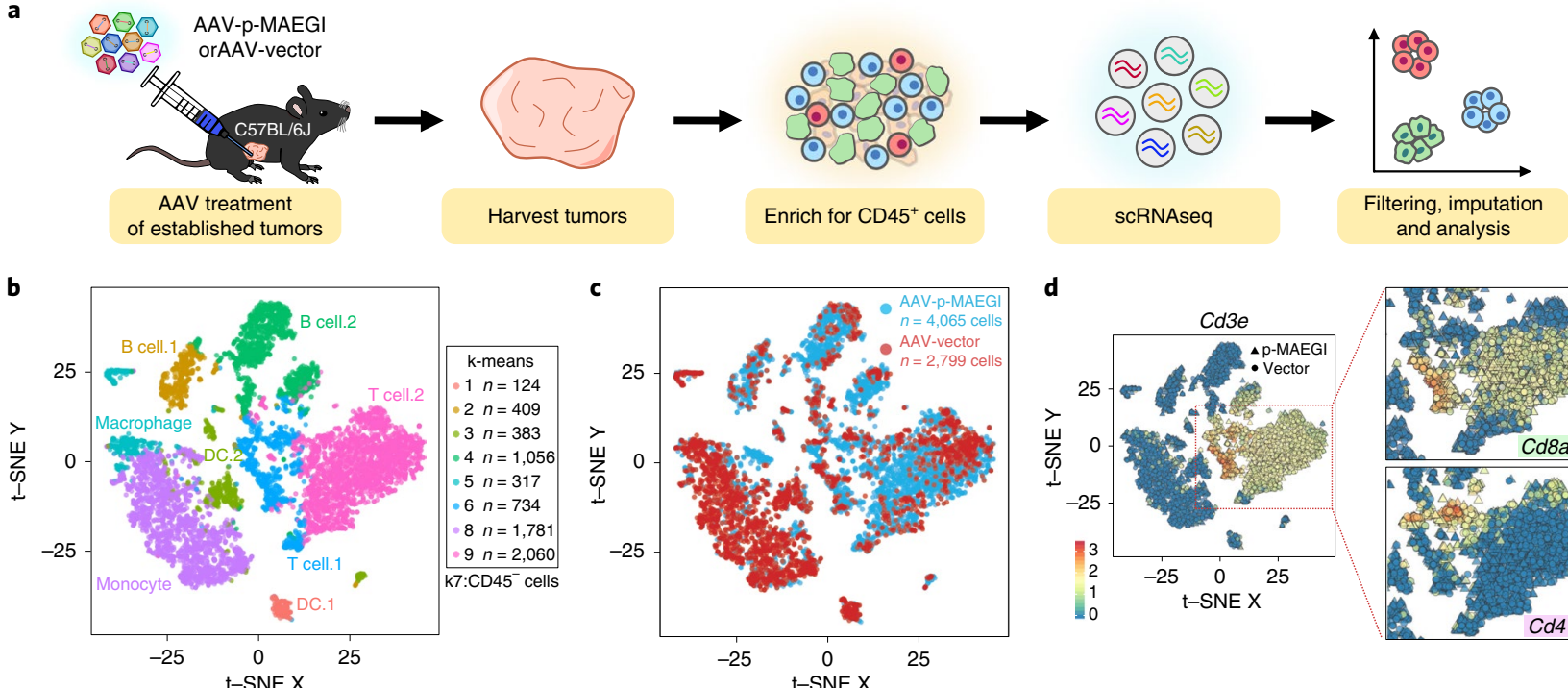

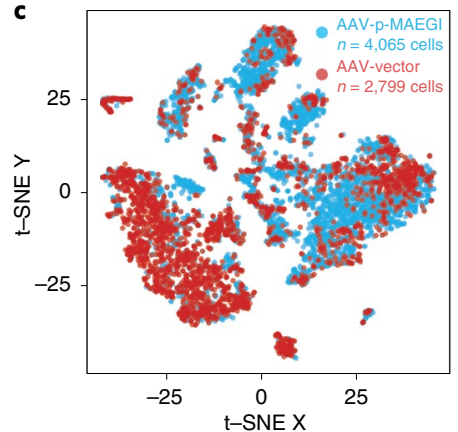

d

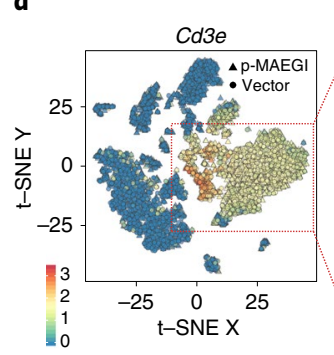

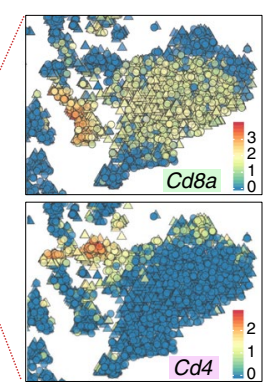
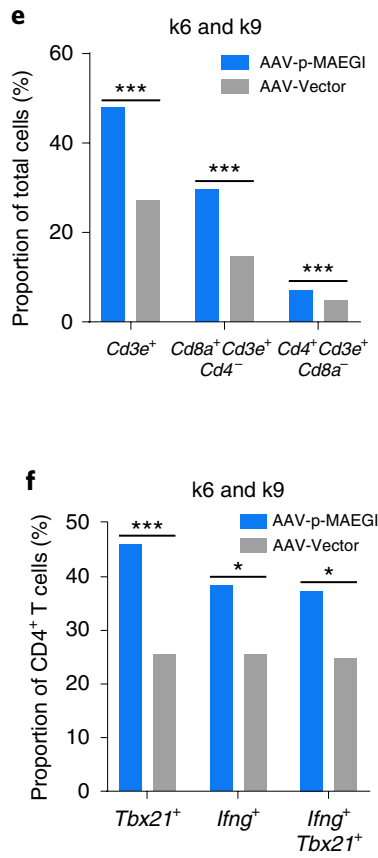

${ }^{8}$

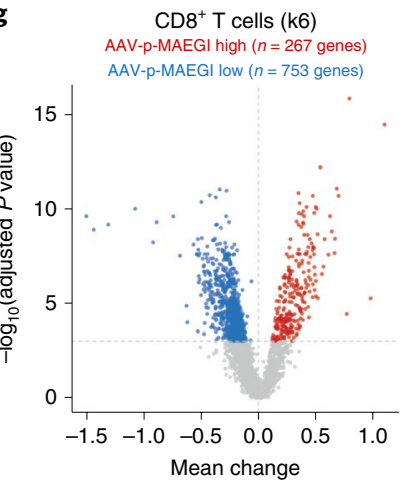

h
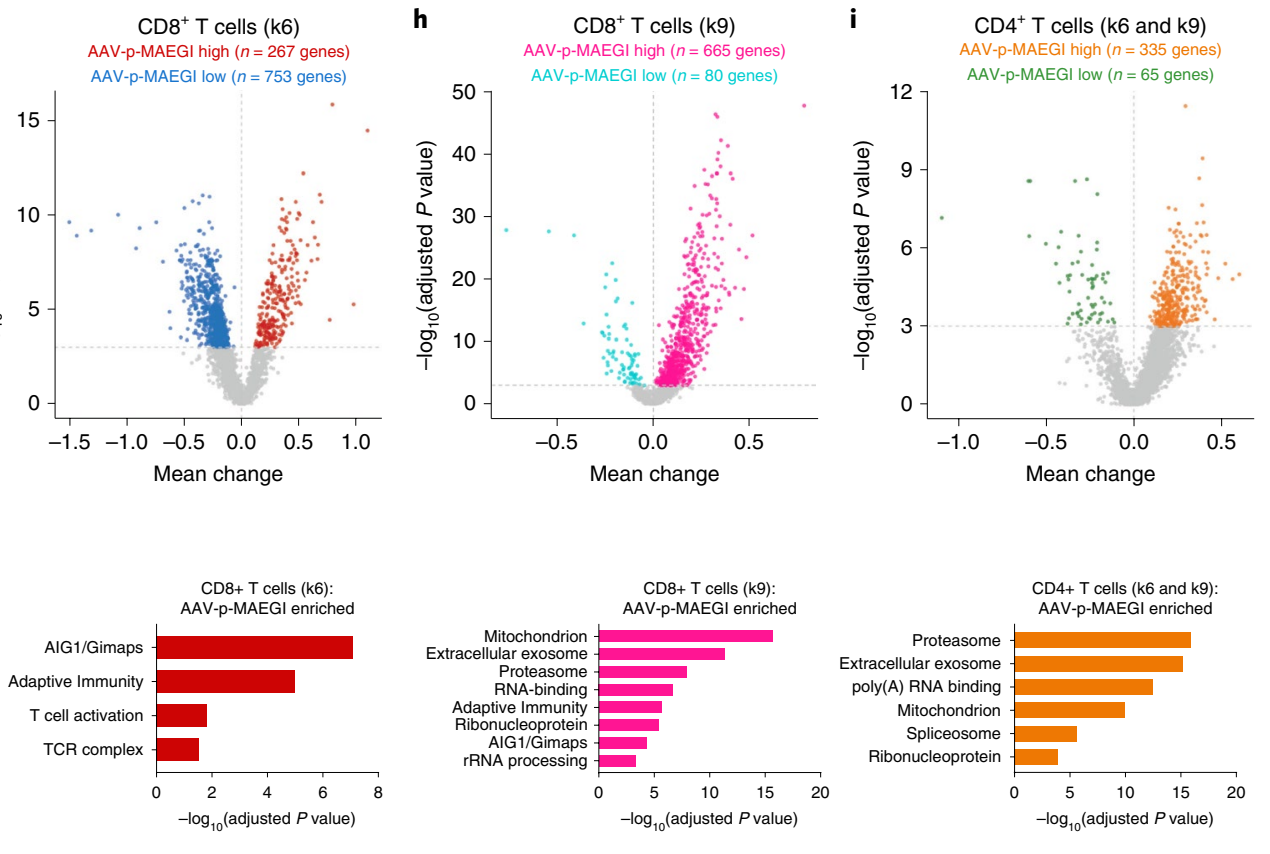

Fig. 7 | Single-cell transcriptomics profiling of immune populations in the tumor microenvironment. a, Schematic of experimental design for scRNA-seq analysis of immune populations. Cells from $n=3$ independent mice (AAV-vector) or $n=3$ mice (AAV-p-MAEGI) were pooled together for scRNA-seq library preparation. b, Scatter plot of t-SNE dimensional reduction, with cells colored by k-means cluster (cell numbers detailed on right). Putative cell types associated with each cluster are annotated. Cluster $7(k 7)$ is composed of CD45- cells and was excluded from further analyses (see Supplementary Fig. 7). c, Scatter plot of t-SNE dimensional reduction as in $\mathbf{b}$, colored by treatment group. The scRNA-seq cell numbers after preprocessing: AAV-pMAEGI, $n=4,065$ cells; AAV-vector, $n=2,799$ cells. $\mathbf{d}$, Identification of T cell populations from scRNA-seq. Left, $t$-SNE dimensional reduction, with cells colored by Cd3e expression. Right, zoomed-in view of clusters k6 and k9, colored by Cd8a expression (top) or Cd4 expression (bottom). e, Quantification of T cell populations in $\mathrm{k} 6$ and $\mathrm{k} 9$, out of total cells (AAV-p-MAEGI, $n=4,065$ cells; AAV-vector, $n=2,799$; two-sided Fisher's exact test: $P<0.0001$ for CD3+ $\mathrm{T}$ cells, CD8 ${ }^{+} \mathrm{T}$ cells and CD4 ${ }^{+} \mathrm{T}$ cells). $\mathbf{f}$, Quantification of putative CD4 ${ }^{+} \mathrm{T}$ cell subpopulations in $\mathrm{k} 6$ and $k 9$ out of total CD4 ${ }^{+} \mathrm{T}$ cells (AAV-p-MAEGI, $n=289$ cells; AAV-vector, $n=133$; two-sided Fisher's exact test: $P<0.0001$ for Tbx21+ cells, $P=0.0111$ for Ifng ${ }^{+}$cells and $P=0.0109$ for Tbx21+lfng ${ }^{+}$cells). $\mathbf{g}-\mathbf{i}$, Top, differential expression volcano plots of CD8 $8^{+} \mathrm{T}$ cells in $\mathrm{k} 6(\mathbf{g}), \mathrm{CD} 8^{+} \mathrm{T}$ cells in $\mathrm{k} 9(\mathbf{h})$ and CD4 $4^{+} \mathrm{T}$ cells in $\mathrm{k} 6$ and k9 (i), comparing AAV-p-MAEGI with AAV-vector ( $n=$ number of differentially expressed genes, assessed using the two-sided, Mann-Whitney test with Benjamini-Hochberg multiple hypothesis correction). Bottom, gene ontology enrichment analysis of upregulated genes in T cells from AAV-p-MAEGI mice, determined using Fisher's exact test with Benjamini-Hochberg multiple hypothesis correction. Error bars: all data points in this figure are presented as mean \pm s.e.m. ${ }^{\star} P<0.05$, ${ }^{\star \star} P<0.01,{ }^{\star \star \star} P<0.001$. Additional supporting data: see Supplementary Fig. 7.

among these putative CD4 ${ }^{+} \mathrm{T}$ cells, AAV-p-MAEGI-treated mice had increased abundance of $\mathrm{Tb} \times 21^{+}$, Ifng $^{+}$and $\mathrm{Tb} \times 21^{+}$Ifng ${ }^{+}$cells, indicative of $\mathrm{T}$ helper type 1 cells (Fig. $7 \mathrm{f}$ and see Supplementary
Table 2). Thus, the single-cell transcriptomic analyses on $\mathrm{CD} 45^{+}$ TIICs reaffirmed prior observations by FACS and TCR-seq that AAV-p-MAEGI promotes $\mathrm{T}$ cell infiltration into tumors. 
To investigate the transcriptomic changes in tumor-infiltrating $\mathrm{T}$ cells after AAV-p-MAEGI, differential expression analyses were performed to compare $\mathrm{T}$ cells from AAV-p-MAEGI- and AAVvector-treated mice. Among the putative $\mathrm{CD}^{+} \mathrm{T}$ cells in $\mathrm{k} 6$ (characterized by higher Gzmb expression compared with k9) (Fig. $7 \mathrm{~g}$ ), 267 upregulated and 753 downregulated genes were found with AAV-p-MAEGI treatment compared with AAV-vector (Fig. $7 \mathrm{~g}$ and see Supplementary Table 2). Gene ontology analysis revealed enrichment of several $\mathrm{T}$ cell signatures within the upregulated gene set, including adaptive immunity, $\mathrm{T}$ cell activation and the TCR complex. The strongest enriched gene set was the AIG1 family of GTPases, particularly Gimap genes (GTPases of immunityassociated protein), the associated proteins of which are involved in $\mathrm{T}$ cell activation and survival ${ }^{33}$. As for the putative $\mathrm{CD}^{+} \mathrm{T}$ cells in k9, 665 genes were upregulated and 80 genes downregulated with AAV-p-MAEGI treatment (Fig. 7h). Enriched gene ontologies in the upregulated gene set included mitochondrion, exosome, proteasome, RNA binding, adaptive immunity, ribonucleoprotein, AIG1/ Gimaps and ribosomal RNA processing. The same analysis was performed on putative $\mathrm{CD} 4^{+} \mathrm{T}$ cells, revealing 335 upregulated and 65 downregulated genes with AAV-p-MAEGI treatment (Fig. 7i). Collectively, the scRNA-seq data reaffirmed that AAV-p-MAEGI treatment increases $\mathrm{T}$ cell infiltration into tumors, and further revealed transcriptomic changes in TILs after MAEGI treatment.

\section{Discussion}

Tumor cells harbor a multitude of mutated, partially truncated or amplified genes that are potentially immunogenic ${ }^{17,34,35}$. However, these mutant products might not be expressed at levels sufficient to elicit an effective T cell-mediated response, and cancer cells often downregulate antigen presentation to escape immune recognition ${ }^{19}$. Several approaches have been described to increase the immunogenicity of tumor cells by in situ manipulation of the tumor microenvironment $^{36-43}$. A key advantage of MAEGI is its versatility, because CRISPR sgRNA libraries can be easily customized to augment the expression of any tumor antigens in a multiplexed manner ${ }^{27,44,45}$, as well as alleged noncoding transcripts, immunomodulatory molecules, cytokines, chemokines or transcription factors, either individually or in combinations.

With high-throughput exome sequencing, personalized immunotherapy targeting patient-specific mutations is now feasible ${ }^{46}$. Synthesis of mutant peptides or transcripts has been used for personal neoantigen vaccine development ${ }^{21-24}$, but the scalability of these approaches is limited. We reasoned that recently developed CRISPRa technologies could radically increase the scale of cancer antigen targeting. By targeting mutant genes for overexpression, MAEGI utilizes host cellular machinery to process and present these abnormal antigens in higher abundance. The data of the present study demonstrate that MAEGI increases target gene expression, increases antigen presentation, increases $\mathrm{T}$ cell effector function, recruits $\mathrm{CD}^{+}$and $\mathrm{CD}^{+} \mathrm{T}$ cells to the tumor microenvironment, and promotes both local and systemic antitumor $\mathrm{T}$ cell responses, thereby eliciting potent antitumor immunity. Distinct from current neoantigen vaccines, AAV-MAEGI represents an orthogonal immunotherapy that directly targets the interplay between tumor cells and the immune microenvironment, inducing longer-term immune memory.

Of note, AAV is now a US Food and Drug Administrationapproved therapeutic transgene vector (see voretigene neparvovec $)^{47,48}$. As a potential viral gene therapy in oncology, administration of AAV-p-MAEGI could be performed to primary tumors, metastatic sites or residual disease as a surgery adjuvant. Importantly, as AAV-MAEGI activates endogenous genes, the acquisition of additional mutations by the tumor, which may have driven relapse by nullifying existing antitumor immunological memory, can still be targeted by MAEGI, as long as the library contains sgRNAs targeting those genes. With a sufficiently diverse sgRNA library, AAV-g-MAEGI or AAV-p-MAEGI can potentially remain efficacious even in the face of continual tumor evolution and immunoediting. It is likely that neutralizing antibodies will be developed against AAV, dCas9 or other components of MAEGI. However, we infer that not all the viruses are neutralized, given our observation that it is important to have consecutive treatments to maintain efficacy. Local high-dose administration may overcome this issue as long as a fraction of cells is transduced, thereby becoming hyper-presenters. Other AAV subtypes and other types of catalytically dead, RNA-guided nucleases can also be used to reduce neutralization.

In summary, in the present study we demonstrated that direct activation of endogenous mutant genes through CRISPRa amplifies the 'nonself' signals of tumor cells, inducing potent antitumor adaptive immunity. The various forms of MAEGI, including AAVg-MAEGI and AAV-p-MAEGI, as well as any future derivatives of endogenous gene activation-based therapies, offer an orthogonal modality of cancer immunotherapy that may serve either as single agents or in concert with other therapeutic modalities. Future clinical translation of MAEGI will require the exclusion of potentially harmful genes when overexpressed, optimization of composition and design, toxicity evaluation in animal models, and investigational new drug-enabling studies, before proceeding to a phase I clinical trial.

\section{Online content}

Any methods, additional references, Nature Research reporting summaries, source data, statements of code and data availability and associated accession codes are available at https://doi.org/10.1038/ s41590-019-0500-4.

Received: 1 July 2019; Accepted: 20 August 2019;

Published online: 14 October 2019

\section{References}

1. Sharma, P., Hu-Lieskovan, S., Wargo, J. A. \& Ribas, A. Primary, adaptive, and acquired resistance to cancer immunotherapy. Cell 168, 707-723 (2017).

2. Herbst, R. S., Morgensztern, D. \& Boshoff, C. The biology and management of non-small cell lung cancer. Nature 553, 446-454 (2018).

3. Rosenberg, S. A. \& Restifo, N. P. Adoptive cell transfer as personalized immunotherapy for human cancer. Science 348, 62-68 (2015).

4. Robert, C. et al. Pembrolizumab versus ipilimumab in advanced melanoma. N. Engl. J. Med. 372, 2521-2532 (2015).

5. Sharma, P. \& Allison, J. P. The future of immune checkpoint therapy. Science 348, 56-61 (2015)

6. Ribas, A. \& Wolchok, J. D. Cancer immunotherapy using checkpoint blockade. Science 359, 1350-1355 (2018).

7. June, C. H., O’Connor, R. S., Kawalekar, O. U., Ghassemi, S. \& Milone, M. C. CAR T cell immunotherapy for human cancer. Science 359, 1361-1365 (2018).

8. Sahin, U. \& Tureci, O. Personalized vaccines for cancer immunotherapy. Science. 359, 1355-1360 (2018).

9. Chen, D. S. \& Mellman, I. Oncology meets immunology: the cancerimmunity cycle. Immunity 39, 1-10 (2013).

10. Chen, D. S. \& Mellman, I. Elements of cancer immunity and the cancerimmune set point. Nature 541, 321-330 (2017).

11. Mellman, I., Coukos, G. \& Dranoff, G. Cancer immunotherapy comes of age. Nature 480, 480-489 (2011).

12. Hamid, O. et al. Safety and tumor responses with lambrolizumab (anti-PD-1) in melanoma. N. Engl. J. Med. 369, 134-144 (2013).

13. Hodi, F. S. et al. Improved survival with ipilimumab in patients with metastatic melanoma. N. Engl. J. Med. 363, 711-723 (2010).

14. Wolchok, J. D. et al. Nivolumab plus ipilimumab in advanced melanoma. N. Engl. J. Med. 369, 122-133 (2013).

15. Topalian, S. L. et al. Safety, activity, and immune correlates of anti-PD-1 antibody in cancer. N. Engl. J. Med. 366, 2443-2454 (2012).

16. Dunn, G. P., Bruce, A. T., Ikeda, H., Old, L. J. \& Schreiber, R. D. Cancer immunoediting: from immunosurveillance to tumor escape. Nat. Immunol. 3, 991-998 (2002).

17. Schumacher, T. N. \& Schreiber, R. D. Neoantigens in cancer immunotherapy. Science 348, 69-74 (2015). 
18. Verdegaal, E. M. et al. Neoantigen landscape dynamics during human melanoma-T cell interactions. Nature 536, 91-95 (2016).

19. Khong, H. T. \& Restifo, N. P. Natural selection of tumor variants in the generation of 'tumor escape' phenotypes. Nat. Immunol. 3, 999-1005 (2002).

20. Spiotto, M. T. et al. Increasing tumor antigen expression overcomes 'ignorance' to solid tumors via crosspresentation by bone marrow-derived stromal cells. Immunity 17, 737-747 (2002).

21. Ott, P. A. et al. An immunogenic personal neoantigen vaccine for patients with melanoma. Nature 547, 217-221 (2017)

22. Sahin, U. et al. Personalized RNA mutanome vaccines mobilize poly-specific therapeutic immunity against cancer. Nature 547, 222-226 (2017).

23. Hilf, N. et al. Actively personalized vaccination trial for newly diagnosed glioblastoma. Nature 565, 240-245 (2019).

24. Keskin, D. B. et al. Neoantigen vaccine generates intratumoral T cell responses in phase Ib glioblastoma trial. Nature 565, 234-239 (2019).

25. Qi, L. S. et al. Repurposing CRISPR as an RNA-guided platform for sequence-specific control of gene expression. Cell 152, 1173-1183 (2013).

26. Gilbert, L. A. et al. CRISPR-mediated modular RNA-guided regulation of transcription in eukaryotes. Cell 154, 442-451 (2013).

27. Konermann, S. et al. Genome-scale transcriptional activation by an engineered CRISPR-Cas9 complex. Nature 517, 583-588 (2015).

28. Chavez, A. et al. Highly efficient Cas9-mediated transcriptional programming. Nat. Methods 12, 326-328 (2015).

29. Tanenbaum, M. E., Gilbert, L. A., Qi, L. S., Weissman, J. S. \& Vale, R. D. A protein-tagging system for signal amplification in gene expression and fluorescence imaging. Cell 159, 635-646 (2014).

30. Zincarelli, C., Soltys, S., Rengo, G. \& Rabinowitz, J. E. Analysis of AAV serotypes 1-9 mediated gene expression and tropism in mice after systemic injection. Mol. Ther. 16, 1073-1080 (2008).

31. Mingozzi, F. \& High, K. A. Therapeutic in vivo gene transfer for genetic disease using AAV: progress and challenges. Nat. Rev. Genet. 12 341-355 (2011).

32. Kirsch, I., Vignali, M. \& Robins, H. T-cell receptor profiling in cancer Mol. Oncol. 9, 2063-2070 (2015).

33. Ciucci, T. \& Bosselut, R. Gimap and T cells: a matter of life or death. Eur. J. Immunol. 44, 348-351 (2014).

34. Martincorena, I. \& Campbell, P. J. Somatic mutation in cancer and normal cells. Science 349, 1483-1489 (2015).

35. Liu, X. S. \& Mardis, E. R. Applications of Immunogenomics to Cancer. Cell 168, 600-612 (2017)

36. van den Boorn, J. G. \& Hartmann, G. Turning tumors into vaccines: co-opting the innate immune system. Immunity 39, 27-37 (2013)

37. Marabelle, A., Tselikas, L., de Baere, T. \& Houot, R. Intratumoral immunotherapy: using the tumor as the remedy. Ann. Oncol. 28, 33-43 (2017).

38. Townsend, S. E. \& Allison, J. P. Tumor rejection after direct costimulation of $\mathrm{CD}^{+} \mathrm{T}$ cells by B7-transfected melanoma cells. Science 259, 368-370 (1993).

39. Chen, L. et al. Costimulation of antitumor immunity by the B7 counterreceptor for the T lymphocyte molecules CD28 and CTLA-4. Cell 71, 1093-1102 (1992).

40. Ribas, A. et al. Oncolytic virotherapy promotes intratumoral $\mathrm{T}$ cell infiltration and improves anti-PD-1 immunotherapy. Cell 170, 1109-1119 (2017)

41. Zamarin, D. et al. Localized oncolytic virotherapy overcomes systemic tumor resistance to immune checkpoint blockade immunotherapy. Sci. Transl. Med. 6, 226ra232 (2014).

42. Suva, M. L. et al. Reconstructing and reprogramming the tumor-propagating potential of glioblastoma stem-like cells. Cell 157, 580-594 (2014)

43. Roulois, D. et al. DNA-Demethylating agents target colorectal cancer cells by inducing viral mimicry by endogenous transcripts. Cell 162, 961-973 (2015)

44. Shalem, O. et al. Genome-scale CRISPR-Cas9 knockout screening in human cells. Science 343, 84-87 (2014).

45. Wang, T., Wei, J. J., Sabatini, D. M. \& Lander, E. S. Genetic screens in human cells using the CRISPR-Cas9 system. Science 343, 80-84 (2014).
46. Robbins, P. F. et al. Mining exomic sequencing data to identify mutated antigens recognized by adoptively transferred tumor-reactive T cells. Nat. Med. 19, 747-752 (2013).

47. Russell, S. et al. Efficacy and safety of voretigene neparvovec (AAV2hRPE65v2) in patients with RPE65-mediated inherited retinal dystrophy: a randomised, controlled, open-label, phase 3 trial. Lancet 390, 849-860 (2017).

48. Bainbridge, J. W. et al. Long-term effect of gene therapy on Leber's congenital amaurosis. N. Engl. J. Med. 372, 1887-1897 (2015).

\section{Acknowledgements}

We thank all members of the Chen laboratory, as well as various colleagues in the Department of Genetics, Systems Biology Institute, Immunobiology Program, BBS Program, Comprehensive Cancer Center, Stem Cell Center and the School of Medicine at Yale, for their assistance and/or scientific discussion. We thank the Center for Genome Analysis, Center for Molecular Discovery, Pathology Tissue Services, Histology Services, High Performance Computing Center, West Campus Analytical Chemistry Core, and West Campus Imaging Core and Keck Biotechnology Resource Laboratory at Yale for technical support. We thank P. Cresswell for assistance with ELISpot. S.C. was supported by Yale SBI/Genetics Startup Fund, National Institutes of Health/National Cancer Institute (NIH/NCI) (grant nos DP2CA238295, R01CA231112, R33CA225498, U54CA209992-8697, RF1DA048811, P50CA196530-A10805, P50CA121974-A08306), Damon Runyon Dale Frey Award (grant no. DFS-13-15), Melanoma Research Alliance (grant nos 412806, 16-003524), St Baldrick's Foundation (grant no. 426685), Breast Cancer Alliance, Cancer Research Institute, American Association for Cancer Research (grant nos 499395, 17-20-01-CHEN), the Mary Kay Foundation (grant no. 017-81), the V Foundation (grant no. V2017-022), Ludwig Family Foundation, DoD (grant no. W81XWH-17-1-0235), Sontag Foundation and Chenevert Family Foundation. C.S.F. was supported by NIH/NCI Cancer Center Support Grant (no. 3P30CA016359). G.W. was supported by CRI Irvington and RJ Anderson Postdoctoral Fellowships. R.D.C., J.P. and M.B.D. were supported by the Yale MSTP training grant from the NIH (grant no. T32GM007205). P.R. was supported by a Yale PhD training grant from NIH (grant no. T32GM007499). X.D. was supported by a C Revson Postdoctoral Fellowship.

\section{Author contributions}

G.W., R.D.C. and S.C. designed the study. G.W. performed most of the experiments in the present study. R.D.C. designed the libraries and analyzed most of the highthroughput data. Z.B., L.Z., Y.E., X.D., M.B.D., L.Y., X.Z., L.S. and H.Y. assisted with the experiments. P.A.R. and J.J.P. assisted with the data analysis. C.S.F. provided clinical insights. S.C. conceived the study, secured funding and supervised the work. G.W. R.D.C. and S.C. prepared the manuscript with input from all authors.

\section{Competing interests}

A patent application has been filed by Yale University related to data in the present study. C.S.F. serves a consulting role for Agios, Bain Capital, Bayer, Celgene, Dicerna, Five Prime Therapeutics, Gilead Sciences, Eli Lilly, Entrinsic Health, Genentech, KEW, Merck, Merrimack Pharmaceuticals, Pfizer, Roche, Sanofi, Taiho and Unum Therapeutics, and also serves as a director for CytomX Therapeutics and owns unexercised stock options for CytomX and Entrinsic Health.

\section{Additional information}

Supplementary information is available for this paper at https://doi.org/10.1038/ s41590-019-0500-4.

Correspondence and requests for materials should be addressed to S.C.

Peer review information Zoltan Fehervari was the primary editor on this article and managed its editorial process and peer review in collaboration with the rest of the editorial team.

Reprints and permissions information is available at www.nature.com/reprints. Publisher's note Springer Nature remains neutral with regard to jurisdictional claims in published maps and institutional affiliations.

(c) The Author(s), under exclusive licence to Springer Nature America, Inc. 2019 


\section{Methods}

Institutional approval. This study has received institutional regulatory approval. All recombinant DNA and biosafety work was performed under the guidelines of Yale Environment, Health and Safety Committee with an approved protocol (Chen-rDNA-15-45). All animal work was performed under the guidelines of Yale University's Institutional Animal Care and Use Committee with approved protocols (Chen-2015-20068, Chen-2018-20068), and was consistent with the Guide for Care and Use of Laboratory Animals, National Research Council, 1996 (Institutional Animal Welfare Assurance no. A-3125-01).

Cell lines. E0771 was from CH3. Pan02 and B16F10 were from ATCC. HEK293FT was from Thermo Fisher Scientific. Various derivative lines were made in the present study as described below. All cell lines tested negative for Mycoplasma spp.

Lentivirus production. Mouse CRISPRa plasmid libraries (lenti-U6-mSAM-EFSPuro) were expanded by electroporation ${ }^{27}$. An estimated library coverage of more than $\times 100$ (>100 colonies per sgRNA) was achieved in electroporation, and the coverage of sgRNAs was subsequently sequence verified by Illumina sequencing. For lentivirus production, $20 \mu \mathrm{g}$ of plasmids of lenti-EF1a-NLS-dCas9-VP64-P2ABlast, lenti-EF1a-MS2-p65-HSF1-P2A-Hygro, lenti-U6-sgRNA(ms2)-EFS-Puro vector or library, together with $10 \mu \mathrm{g}$ of pMD2.G and $15 \mu \mathrm{g}$ of psPAX2, were cotransfected into HEK-293FT cells in a $150-\mathrm{mm}$ dish at $80-90 \%$ confluency using $130 \mu \mathrm{g}$ of polyethyleneimine (PEI) as the transfection reagent. Then $6-12 \mathrm{~h}$ later, the medium was replaced by fresh Dulbecco's minimal essential medium $+10 \%$ fetal bovine serum (FBS). Virus supernatant was collected $48 \mathrm{~h}$ and $72 \mathrm{~h}$ posttransfection, centrifuged at $1500 \mathrm{~g}$ for $10 \mathrm{~min}$, passed through a $0.45-\mu \mathrm{m}$ filter to remove the cell debris, aliquoted and stored at $-80^{\circ} \mathrm{C}$. Library virus was titrated by infecting E0771 cells, followed by the selection under $5 \mu \mathrm{g} \mathrm{ml}^{-1}$ of puromycin.

Antigen presentation assay using an OVA-expressing cell line. A lentiviral mChOva-expressing vector (Lenti-pGK-mCherry-2A-Ova-WPRE; Addgene, no. 129600) was generated via Gibson Assembly. E0771 cells were then transduced using mChOva-expressing lentiviruses. Then $2-3 \mathrm{~d}$ post-transduction, $\mathrm{mChOva-}$ expressing cells (E0771-mChOva) were FACS sorted on a BD FACSAria. OVA expression was further confirmed by staining these cells with antibody specific to the complex of SIINFEKL peptide bound to MHC-I (APC anti-mouse $\mathrm{H}-2 \mathrm{~Kb}$ bound to SIINFEKL antibody). Then, E0771-mChOva cells were transduced with lentiviral lenti-EF1a-NLS-dCas9-VP64-2A-Blast and lenti-EF1a-MS2-p65HSF1-2A-Hygro, followed by $7 \mathrm{~d}$ of selection under the pressure of $10 \mu \mathrm{g} \mathrm{ml}^{-1}$ of blasticidin and $500 \mathrm{~g} \mathrm{~m} \mathrm{ml}^{-1}$ of hygromycin to generate cells stably expressing dCas9VP64 and MS2-P65-HSF1 (E0771-mChOva-dCa9-MPH).

To activate the expression and thus the presentation of mChOva, PGKpromoter-targeting sgRNAs were designed and cloned into Lenti:U6-sgRNA(ms2)EFS-Puro and AAV:U6p-sgSapI-EF1a-MS2-p65-HSF1-WPRE; Addgene, no. 129599) vector. After lentivirus or AAV production, E0771-mChOva-dCa9$\mathrm{MPH}$ cells were infected with these lentiviruses or AAVs to activate mChOva transcription. Then 3-7 d after viral transduction, the cell surface of SIINFEKL $\mathrm{H}-2 \mathrm{~Kb}$ was stained with $\mathrm{PE}$ anti-mouse $\mathrm{H}-2 \mathrm{~Kb}$ bound to SIINFEKL antibody (AAV experiments) or APC anti-mouse $\mathrm{H}-2 \mathrm{~Kb}$ bound to SIINFEKL antibody (lentiviral experiments). The genomic mean intensity of APC-SIINFEKL-H-2Kb/ PE-SIINFEKL-H-2 $\mathrm{K}^{\mathrm{b}}$ was used to determine cell surface presentation of SIINFEKL.

Co-culture of MAEGI-treated cells and effector T cells. SIINFEKL-H-2K $\mathrm{K}^{\mathrm{b}}$ specific naive CD8 T cells were isolated from OT-I mice using a naive mouse CD8 $\mathrm{T}$ cell kit (Miltenyi). Before use, the plate was coated with anti-CD3 $\left(5 \mu \mathrm{g} \mathrm{ml}^{-1}\right.$ in PBS) at $37^{\circ} \mathrm{C}$ for $1 \mathrm{~h}$ and then washed twice with PBS. After this, naive $\mathrm{T}$ cells were cultured in the anti-CD3-coated plate using cRPMI (RPMI$1640+10 \% \mathrm{FBS}+2 \mathrm{mM}$ L-glutamine $+49 \mathrm{nM} 2$-mercaptoethanol $+100 \mathrm{U}$ penicillin-streptomycin) supplemented with $1 \mu \mathrm{g} \mathrm{ml}^{-1}$ of anti-CD28, $2 \mathrm{ng} \mathrm{ml}^{-1}$ of recombinant mouse interleukin (IL)-2 and $2 \mathrm{ng} \mathrm{ml}^{-1}$ of recombinant mouse IL-12 $3 \mathrm{~d}$ later, the effector T cells were cultured in cRPMI supplemented with $2 \mathrm{ng} \mathrm{ml}^{-1}$ of recombinant mouse IL-2 and $5 \mathrm{ng} \mathrm{ml}^{-1}$ of recombinant mouse IL-7 for another 2 d. E0771-mChOva-dCa9-MPH cells transduced with vector or PGK-targeted sgRNAs were seeded in 24 -well plates at a concentration of $2 \times 10^{5}$ cells per well; 2-4h later, OT-1 T cells were counted, resuspended in cRPMI supplemented with IL-2, and added to tumor cell-seeded plates at ratios of effector:tumor $(\mathrm{E}: \mathrm{T})=0.5$ or 1. For killing and apoptosis analysis, the effector T cells and E0771 cells were co-cultured for $24 \mathrm{~h}$. All the samples were collected and stained with anti-CD8a Apc/Cy7 to exclude the T cells. The dead and apoptotic cancer cells were analyzed using PE Annexin V Apoptosis Detection Kit with 7-AAD. For interferon detection, $5 \mu \mathrm{g} \mathrm{ml}^{-1}$ Brefeldin A was added and co-cultured for $3 \mathrm{~h}$. Then, samples were collected and stained with anti-CD45 PerCP/Cy5.5 and anti-CD8a Apc/Cy7 for $30 \mathrm{~min}$. After washing twice, the cells were fixed with BD Cytofix buffer for $10 \mathrm{~min}$ on ice and washed with Cytoperm/Wash Buffer. Cells were then subjected to intracellular staining with anti-IFN- $\gamma$ APC for 30 min on ice. After washing once with Cytoperm/Wash Buffer, the samples were resuspended in MACS Buffer and analyzed via flow cytometry.
Generation of mSAM library- and vector- transduced cells. E0771 cells stably expressing dCas9-VP64 and MS2-P65-HSF1 (E0771-dCas9-VP64-MPH) were generated by transducing lentiviral lenti-EF1a-NLS-dCas9-VP64-2A-Blast and lenti-EF1a-MS2-p65-HSF1-2A-Hygro into E0771 cells, followed by $7 \mathrm{~d}$ of selection under the pressure of $10 \mu \mathrm{g} \mathrm{ml}^{-1}$ of blasticidin and $500 \mu \mathrm{g} \mathrm{ml}^{-1}$ of hygromycin. E0771-dCas9-VP64-MPH cells were then infected with lentiviral mSAM library or vector to obtain mSAM-transduced E0771 (E0771-SAM) or vector-transduced E0771 (E0771-vector). Some $1 \times 10^{8}$ cells were initially infected by the lentiviral mSAM sgRNA library at a calculated multiplicity of infection of 0.2 , with a minimal representation of $\times 200$ transduced cells per sgRNA, as described previously $y^{27}$. Lentivirus-infected cells were cultured at $37^{\circ} \mathrm{C}$ for more than $1 \mathrm{~d}$ before replacing with $5 \mu \mathrm{g} \mathrm{ml}^{-1}$ of puromycin-containing medium, and the transduced cells were drug selected for $7 \mathrm{~d}$ before use.

Sequencing confirmation of SAM sgRNA library representation. Librarytransduced cells were subjected to genomic DNA (gDNA) extraction using standard molecular biology protocols. The sgRNA library readout was performed using a two-step PCR strategy, in which the first PCR included enough gDNA to preserve full library complexity and the second PCR added appropriate sequencing adapters to the products from the first PCR.

\section{PCR1 primers. Forward:}

AATGGACTATCATATGCTTACCGTAACTTGAAAGTATTTCG

Reverse:

CTTTAGTTTGTATGTCTGTTGCTATTATGTCTACTATTCTTTCCC

PCR was performed using Phusion Flash High Fidelity Master Mix (PF) (Thermo Fisher Scientific). For reactions using PF, in PCR1, the thermocycling parameters were: $98^{\circ} \mathrm{C}$ for $1 \mathrm{~min}, 16-20$ cycles (of $98^{\circ} \mathrm{C}$ for $1 \mathrm{~s}, 62^{\circ} \mathrm{C}$ for $5 \mathrm{~s}, 72^{\circ} \mathrm{C}$ for $30 \mathrm{~s}$ ) and $72^{\circ} \mathrm{C}$ for $2 \mathrm{~min}$. In each PCR1 reaction, $3 \mu \mathrm{g}$ of total gDNA was used. A total of 8-12 PCR1 reactions was used to capture the full representation of the library in the cells. PCR1 products for each biological sample were pooled and used for amplification with barcoded second PCR primers.

Barcoding second PCR1 primers used. SF5: AATGATACGGCGACCACCGAGATCTACACTCTTTCCCTACACGACGCTCTTCCGATCTTCGAT-

CGTTACCATCTTGTGGAAAGGACGAAACACCG

SF6: AATGATACGGCGACCACCGAGATCTACACTC-

TTTCCCTACACGACGCTCTTCCGATCTATCGATTCCTTG-

GTTCTTGTGGAAAGGACGAAACACCG

SR1: CAAGCAGAAGACGGCATACGAGATAAGTAGAGGTGACTGGAGTTCAGACGTGTGCTCTTCCGATCTTTCTACTATTCTTTCCCCTGCACTGT

SR2: CAAGCAGAAGACGGCATACGAGATACACGATCGTGACTGGAGT-

TCAGACGTGTGCTCTTCCGATCTATTCTACTATTCTTTCCCCTGCACTGT

SR3: CAAGCAGAAGACGGCATACGAGATCGCGCGGTGTGACTGGAGT-

TCAGACGTGTGCTCTTCCGATCTGATTCTACTATTCTTTCCCCTGCACTGT

SR4: CAAGCAGAAGACGGCATACGAGATCATGATC-

GGTGACTGGAGTTCAGACGTGTGCTCTTCCGATCTCGAT-

TCTACTATTCTTTCCCCTGCACTGT

SR5: CAAGCAGAAGACGGCATACGAGATCGTTACC-

AGTGACTGGAGTTCAGACGTGTGCTCTTCCGATCTCGAT-

CTCTACTATTCTTTCCCCTGCACTGT

SR6: CAAGCAGAAGACGGCATACGAGATTCCTTGGTGTGACTGGAGTT

CAGACGTGTGCTCTTCCGATCTTTCTACTATTCTTTCCCCTGCACTGT

For reactions using Phusion Flash, in PCR2, the thermocycling parameters were: $98^{\circ} \mathrm{C}$ for $1 \mathrm{~min}, 18-24$ cycles (of $98^{\circ} \mathrm{C}$ for $1 \mathrm{~s}, 60^{\circ} \mathrm{C}$ for $5 \mathrm{~s}, 72^{\circ} \mathrm{C}$ for $30 \mathrm{~s}$ ) and $72^{\circ} \mathrm{C}$ for $2 \mathrm{~min}$. PCR 2 products were pooled and then normalized for each biological sample before combining uniquely barcoded, separate biological samples. The pooled product was then gel purified from a $2 \%$ E-gel EX (Life Technologies) using the QIAquick Gel Extraction Kit (Qiagen). The purified pooled library was then quantified using a gel-based method with the Low-Range Quantitative Ladder Life Technologies, dsDNA High-Sensitivity Qubit (Life Technologies), BioAnalyzer (Agilent) and/or quantitative (q)PCR. Diluted libraries with 5-20\% PhiX were sequenced with MiSeq, HiSeq 2500 or HiSeq 4000 systems (Illumina).

Raw, single-end, fastq-read files were filtered and demultiplexed using Cutadapt $^{49}$. To remove sgRNA scaffold sequences downstream (that is $3^{\prime}$-end) of the sgRNA spacer sequences, the following command was used: cutadapt --discard-untrimmed -a GTTTTAGAGCTAGGCCAAC. As the forward PCR primers used to read out sgRNA representation were designed to have a variety of barcodes to facilitate multiplexed sequencing, these filtered reads were then demultiplexed with the following settings: cutadapt -g file:fbc.fasta --no-trim, where fbc.fasta contained the possible barcode sequences within the forward primers. Finally, to remove non-sgRNA sequences upstream (that is $5^{\prime}$-end) of the sgRNA spacers, the following command was used: cutadapt --discard-untrimmed -g GTGGAAAGGACGAAACACCG. Through this procedure, the raw fastq read files were trimmed to the 20-basepair (bp) sgRNA spacer sequences. The 20-bp sgRNA spacer sequences from each demulitplexed sample were mapped for the sgRNA spacers to the mSAM library using Bowtie v.1.1.2 (ref. ${ }^{50}$ ): bowtie -v 2 --suppress 4,5,6,7 --chunkmbs 2000 -best. Using the resultant mapping output, the number of reads that had mapped to each sgRNA within the library was quantified. 
Mice. Mice of both sexes, between the ages of 5 and 12 weeks, were used for the study. Various animals were used in this study; 5- to 10-week-old C57BL/6J mice were used for experiments unless otherwise specified. Female mice were used for breast cancer (E0771) models. Male mice were used for pancreatic adenocarcinoma (Pan02) models. A mix of both male and female mice was used for melanoma (B16F10) models. All animals were housed in standard, individually ventilated, pathogen-free conditions, with a $12 \mathrm{~h}: 12 \mathrm{~h}$ or a $13 \mathrm{~h}: 11 \mathrm{~h}$ light cycle, at room temperature $\left(21-23^{\circ} \mathrm{C}\right)$ and $40-60 \%$ relative humidity. Sample size determination (that is, number of mice per treatment group) was based on similar tumor models in the field. When a cohort of animals was to receive different treatments, animals were randomized by: (1) randomly assigning littermates to different groups before treatment, maximizing the evenness or representation of mice from different cages in each group; (2) group animals before treatment so that each group has even distribution of initial tumor sizes; and/or (3) random assignment of mice to minimize the effect of litter, and small differences in age, cage or housing position, where applicable.

Tumorigenesis of E0771-SAM in C57BL/6J, Nude (Foxn $1^{n u}$ ) or Rag1 ${ }^{-/-}$mice. E0771-SAM or E0771-vector tumor cells, $5 \times 10^{6}$, were injected into the orthotopic mammary fat pad of syngeneic 5- to 8-week-old female C57BL/6J, Nude (Foxn $1^{n u}$ ) or $\mathrm{Ragl}^{-/-}$mice. Tumor sizes were measured every 3-4 $\mathrm{d}$ using calipers of the three diameters, and sizes were calculated using the formula: volume $=\pi / 6 x y z$ (where $x$ is tumor length, $y$ is tumor width, and $z$ is tumor depth). The statistical significance of all tumor growth curves in the present study was assessed using two-way analysis of variance (ANOVA), jointly considering the effect of treatment and the passage of time on tumor growth. For CD4 and CD8 T cell depletion, $200 \mu \mathrm{g}$ of anti-CD4 (GK1.5, BioXcell) and $200 \mu \mathrm{g}$ of anti-CD8 (YTS 169.4) were intraperitoneally injected into tumor-bearing C57BL/6J mice at dpi 7 and dpi 14 . The successful depletion of CD8 T cells was confirmed by isolating peripheral blood mononuclear cells from mice at dpi 14 and 21, and analyzing the CD8 ${ }^{+} \mathrm{T}$ cell population using flow cytometry.

ELISpot assay. IFN- $\gamma$ ELISpot mouse kits (BD Biosciences) were used according to the manufacturer's instructions. Briefly, 96-well filtration plates were coated overnight at $4{ }^{\circ} \mathrm{C}$ with IFN- $\gamma$-capturing monoclonal antibody; the plates were then washed and blocked with RPMI-1640 medium containing $10 \%$ FBS for $2 \mathrm{~h}$ at room temperature. Splenocytes and TIICs isolated from different treatment groups were counted and resuspended in 10\% FBS-supplemented RPMI-1640. Splenocytes and TIICs were seeded into the 96-well filtration plate at concentrations of $1 \times 10^{6}$ cells per well and $2.5 \times 10^{4}$ cells per well, respectively. After mitomycin treatment, $5 \times 10^{4} \mathrm{C} 57 \mathrm{BL} / 6 \mathrm{~J}$ fat pad-derived primary cells or E0771 tumor cells were added as different stimulators to stimulate the IFN- $\gamma$ secretion. The plates were cultured for approximately $45 \mathrm{~h}$ at $37^{\circ} \mathrm{C}$ and $5 \% \mathrm{CO}_{2}$. The co-culture were stopped by soaking in deionized water, and washing three times with PBS with Tween 20; the plates were incubated with biotinylated detection antibody at room temperature for $2 \mathrm{~h}$. Then, plates were incubated with horseradish peroxidase (HRP)-conjugated streptavidin at room temperature for $1 \mathrm{~h}$ after washing three times with PBS with Tween 20. Spots were revealed using an AEC substrate reagent kit (BD Bioscience) at room temperature, and counted using an Immunospot Reader (Cellular Technology). The statistical significance was assessed using a two-tailed, unpaired, Welch's $t$-test.

Generation of AAV-MAEGI. An AAV version of the CRISPRa vector (AAVCRISPRa vector, that is U6p-sgSapI-EF1a-MS2-p65-HSF1-sPA; Addgene, no. 129602), was generated by restriction cloning and Gibson assembly. The sgRNA libraries of mSAM were cloned into the above CRISPRa plasmid by linearization with SapI digestion and Gibson assembly. The purification and electroporation of Gibson products into Endura electrocompetent cells were performed as previously described $^{51}$, with at least $\times 100$ coverage of colonies represented per sgRNA. AAV was produced by co-transfecting HEK-293FT cells with the above AAV plasmids of CRISPRa vector or library (AAV-mSAM), AAV2, AAV9 or AAV-DJ serotype plasmid and helper plasmid PDF6, to generate AAV-g-MAEGI. Briefly, 5.2 $\mu \mathrm{g}$ of AAV-Vector, AAV-mSAM or other AAV-sglib plasmid, $8.7 \mu$ g of plasmid AAV9 or AAV-DJ serotype, and $10.4 \mu \mathrm{g}$ of pDF6 were mixed with PEI, at room temperature for 10-15 min before adding them drop-wise into HEK-293FT cells in a 150$\mathrm{mm}$ dish at $80-90 \%$ confluency. Replicates collected from multiple dishes were pooled to enhance production yield. Cells were collected $72 \mathrm{~h}$ post-transfection. For AAV purification, chloroform (1:10 by volume) was added and the mixture was vigorously shaken for $1 \mathrm{~h}$ at $37^{\circ} \mathrm{C}$. $\mathrm{NaCl}$ was added to a final concentration of $1 \mathrm{M}$, and the mixture was shaken until dissolved and then pelleted at $20,000 \mathrm{~g}$ and $4^{\circ} \mathrm{C}$ for $15 \mathrm{~min}$. The aqueous layer was discarded while the chloroform layer was transferred to another tube. PEG 8000 was added to $10 \%(\mathrm{w} / \mathrm{v})$ and shaken until dissolved. The mixture was incubated at $4^{\circ} \mathrm{C}$ for $1 \mathrm{~h}$ and then spun at $20,000 \mathrm{~g}$ and $4^{\circ} \mathrm{C}$ for $15 \mathrm{~min}$. The supernatant was discarded and the pellet resuspended in Dulbecco's phosphate-buffered saline $+\mathrm{MgCl}_{2}$, treated with Benzonase (Sigma) and incubated at $37^{\circ} \mathrm{C}$ for $30 \mathrm{~min}$. Chloroform (1:1 by volume) was then added, shaken and spun down at $12,000 \mathrm{~g}$ and $4^{\circ} \mathrm{C}$ for $15 \mathrm{~min}$. The aqueous layer was isolated and passed through a $100 \mathrm{kDa}$ MWCO (Millipore). The concentrated solution was washed with PBS and the filtration process repeated. Genomic copy number (GC) of AAV was determined by real-time qPCR using custom Taqman assays (Thermo Fisher Scientific) targeted to the engineered U6 promoter.
Therapeutic testing of AAV-g-MAEGI in syngeneic tumor models. Syngeneic orthotopic breast tumors were established by transplanting $2 \times 10^{6}$ E0771-dCas9VP64 cells into the mammary fat pad of 5- to 8-week-old female C57BL/6J mice. Then, 4, 10, 14 and $21 \mathrm{~d}$ after transplantation, 5-10 $\times 10^{10}$ GCs of AAV-g-MAEGI, $\mathrm{AAV}$-vector or PBS were injected intratumorally into tumor-bearing mice. For the B16F10 melanoma model, $5 \times 10^{5} \mathrm{dCas} 9$-VP64-expressing cancer cells were injected subcutaneously into the flank of C57BL/6J mice; 7, 10, 14 and $20 \mathrm{~d}$ after transplantation, $5 \times 10^{10}-10 \times 10^{10} \mathrm{GCs}$ of AAV-g-MAEGI, AAV-vector or PBS were intratumorally administered to tumor-bearing mice. For the pancreatic tumor model, $2 \times 10^{6}$ Pan02-dCas9-VP64 cancer cells were injected subcutaneously into the flank of C57BL/6J mice. Then, 5, 13, 21 and $35 \mathrm{~d}$ after transplantation, $5-10 \times 10^{10} \mathrm{GCs}$ of AAV-g-MAEGI, AAV-vector or PBS were intratumorally administered to tumor-bearing mice. Tumors were measured every 3-4 d using calipers and the sizes were calculated with the formula: volume $=\pi / 6 x y z$. Two-way ANOVA was used to compare growth curves between treatment groups.

Isolation of splenocytes and TILs. Syngeneic breast tumors were established by orthotopically transplanting $2 \times 10^{6}$ E0771-dCas9-VP64 cells into the mammary fat pad of 5- to 8-week-old female C57BL/6J mice. Tumor-bearing mice were randomly assembled into different treatment groups and treated with various reagents where applicable, as described in the captions. Mice were euthanized at 9, 19,29 or 36d post-transplantation as indicated, and tumors and spleens were collected and kept in ice-cold 2\% FBS. For spleens, they were placed in ice-cold 2\% FBS and mashed through a $100-\mu \mathrm{m}$ filter. Splenocytes were washed once with $2 \%$ FBS. Tumors were minced into 1 - to $3-\mathrm{mm}$ size pieces using a scalper and then digested using $100 \mathrm{U} \mathrm{ml}^{-1}$ Collagenase IV for $30-60 \mathrm{~min}$ while stirring at $37^{\circ} \mathrm{C}$. Tumor suspensions were filtered twice through a $100-\mu \mathrm{m}$ cell strainer, and once through a $40-\mu \mathrm{m}$ cell strainer to remove large bulk masses. Red blood cells were lysed with $1 \mathrm{ml}$ ACK Lysis Buffer (Lonza) per spleen by incubating 2-5 min at room temperature, which was followed by dilution with $10 \mathrm{ml} 2 \% \mathrm{FBS}$ and a pass through a $40-\mu \mathrm{m}$ filter. Splenocytes were resuspended in 2\% FBS buffer, counted for flow cytometry staining or RNA isolation. Single-cell suspensions of tumors were used for flow cytometry staining, further FACS sorting or Ficoll purification to obtain TILs. For Ficoll-Paque purification, single-cell suspensions at a density of $\sim 10^{7}$ cells ml ${ }^{-1}$ were carefully layered on to Ficoll-Paque medium (GE Healthcare) and centrifuged at $400 \mathrm{~g}$ for $30 \mathrm{~min}$. Cells at the interface were carefully collected, and washed twice with $2 \%$ FBS, counted and used for RNA isolation or flow cytometry staining.

Flow cytometry. All antibodies for flow cytometry were purchased from Biolegend or eBiosciences. Single-cell suspensions from tumors or spleens were prepared using a gentleMACS tissue dissociation system. The panels of antibodies used in the flow cytometry staining are as follows: panel 1: anti-CD45.2-APC/Cy7, anti-CD3-PE, anti-CD4-FITC, anti-CD8a-APC; panel 2: anti-CD45-PerCP/ Cy5.5, anti-CD3-FITC, anti-CD4-PE/Cy7, or anti-CD8-APC/Cy7; or panel 3: anti-CD45-APC/Cy7, anti-I-A/I-K-PerCP/Cy5.5, anti-CD11b-FITC, anti-CD11cPE/Dazzle594; anti-Ly6c-APC; or anti-F4/80-PE, anti-CD24-PE/Cy7. All flow antibodies were used at 1:100 dilutions for staining unless otherwise noted. For surface staining, cells were blocked with anti-Fc receptor anti-CD16/CD32, and then stained with surface marker antibodies in a staining buffer of $2 \%$ FBS in PBS on ice for $30 \mathrm{~min}$. Samples were washed twice with $2 \%$ FBS in PBS before analysis. For intracellular staining, eBioscience Intracellular Fixation \& Permeabilization Buffer Set was used to fix and permeabilize cells following the manufacturer's instructions. Briefly, after the staining of surface makers, cells were resuspended in $100 \mu \mathrm{l}$ Fixation/Permeabilization working solution, and incubated on ice for $10 \mathrm{~min}$ before washing with $1 \times$ Permeabilization buffer by centrifugation at $600 g$ for $5 \mathrm{~min}$. Then the cell pellet was resuspended in $50 \mu \mathrm{l}$ of $1 \times$ permeabilization buffer with anti-Fc receptor anti-CD16/CD32, and incubated on ice for $10 \mathrm{~min}$, before adding $50 \mu \mathrm{l}$ of $2 \times$ intracellular staining antibodies and incubating on ice for $30 \mathrm{~min}$. After staining, cells were centrifuged at $600 \mathrm{~g}$ for $5 \mathrm{~min}$, and washed twice with staining buffer before being analyzed or sorted on a BD FACSAria. The data were analyzed using FlowJo software (v.9.9.4 or v.10.3). A previously reported strategy was used to define the populations of monocytes, neutrophils, dendritic cells and macrophages in tumors ${ }^{52}$. Antibodies used for flow cytometry are listed in Supplementary Table 1. Statistical significance was assessed using the two-tailed, Mann-Whitney test.

FACS isolation of $\mathrm{CD}^{+} 5^{+}$cells from tumors. Single tumor cell suspensions were prepared using the gentleMACS system with the method described above. Tumor cells were blocked using anti-Fc receptor anti-CD16/CD32. Live cells were distinguished from dead cells in flow cytometry by staining with a LIVE/DEAD Fixable Near-IR Dead Cell Stain Kit, following the manufacturer's instructions. Cells at a density of $10^{7} \mathrm{ml}^{-1}$ were stained with dimethylsulfoxide-dissolved live/ dead staining dye and PerCP/Cy5.5 or APC-conjugated CD45 antibody in $\mathrm{PBS}+2 \% \mathrm{FBS}$, and incubated on ice for $30 \mathrm{~min}$. Stained cells were washed three times before being analyzed and FACS isolated on a BD FACSAria. TIICs were isolated by gating on $\mathrm{PerCP} / \mathrm{Cy} 7^{+} \mathrm{APC} / \mathrm{Cy} 7^{-}$or $\mathrm{APC}+\mathrm{APC} / \mathrm{Cy} 7^{-}$populations. Sorted TIICs were counted, and used for scRNA-seq and ELISpot analysis.

Immune cell profiling by scRNA-seq. FACS sorted cells were collected in $\mathrm{PBS}+2 \% \mathrm{FBS}$ and concentrated by centrifugation at $400 \mathrm{~g}$ for $5 \mathrm{~min}$. Cell numbers 
and viabilities were assessed by trypan blue staining and analysis in Countess II FL Automated Cell Counter (Thermo Fischer Scientific). The 10,000 CD45 TIICs isolated from tumors were used for scRNA-seq library prep by following the protocol from 10x Genomics.

ScRNA-seq data processing. Pan-immune cell, scRNA-seq data were preprocessed using both established pipelines and custom scripts. Briefly, raw Illumina data files were processed by Cell Ranger 1.3 (10x Genomics) ${ }^{53}$, using cellranger mkfastq to wrap Illumina's bcl2fastq to correctly demultiplex sequencing samples, and to convert barcode and read data to FASTQ files. Then, cellranger count was used to take FASTQ files and perform alignment to the mouse genome $(\mathrm{mm} 10)^{54}$, filtering and UMI counting. Cells with fewer than 2,000 UMI counts were subsequently filtered from analysis, leaving a final set of 7,863 cells for further analysis. Next, imputation was performed using $\mathrm{ALRA}^{55}$, which is a conservative strategy for distinguishing technical 'dropout' zeros from true biological zeros. After log normalization, the 27,998 genes/features were additionally filtered using a flat cutoff metric such that genes with variance $<0.1$ were excluded, leaving 7,632 genes. Using the final normalized and processed dataset above, $t$-distributed stochastic neighbor embedding ( $t$-SNE) dimension reduction was performed ${ }^{56,57}$. Individual data-points were colored, based on either the treatment condition for each cell or unbiased k-means clustering performed on the normalized dataset. Differential expression analyses between clusters and/or treatment conditions were performed using a nonparametric, two-tailed, Mann-Whitney test, filtering for genes with variance $>0.1$ across the cell population of interest. A multiple hypothesis correction was performed using the Benjamini-Hochberg method. Differentially expressed genes were defined as having a Benjamini-Hochbergadjusted $P<0.001$. A cutoff of 0 was used to determine whether cells expressed a particular gene of interest. The statistical significance of differences in cell type proportions between AAV-p-MAEGI and AAV-vector conditions was assessed using a two-tailed, Fisher's exact test. Gene ontology analysis was performed using DAVID ${ }^{58}$

RNA extraction, reverse transcription and qPCR. RNA in cells, splenocytes and TILs was extracted using TRIzol Reagent (Invitrogen) by following standard RNA extraction protocols. The first-strand complementary DNA of RNA was synthesized using SuperScript IV Reverse Transcriptase (Invitrogen). After normalizing the concentrations of cDNA with nuclease-free water, qPCR was performed by adding designated Taqman probe of interested genes, and glyceraldehyde 3-phosphate dehydrogenase was used as an internal positive control.

Western blot. E0771, E0771-dCas9-VP64 and E0771-dCas9-VP64-MPH cells in six-well plates were washed twice with ice-cold PBS before lysis with $1 \times$ radioimmunoprecipitation assay buffer, which was kept on ice for $15 \mathrm{~min}$. Cell lysates were centrifuged at $12,000 \mathrm{~g}$ for $15 \mathrm{~min}$ at $4{ }^{\circ} \mathrm{C}$ and protein-containing supernatant was collected. Protein concentration was measured using a standard Bradford assay (Bio-Rad) and $20 \mu \mathrm{g}$ of protein in each sample was loaded on to a sodium dodecylsulfate/polyacrylamide electrophoresis gel. After electrophoresis, proteins separated in the gel were transferred on to nitrocellulose membranes. The membranes were blocked at room temperature for $1 \mathrm{~h}$ using $5 \%$ skimmed milk in Tris-buffered saline + Tween 20 , followed by incubation with primary antibody in $4{ }^{\circ} \mathrm{C}$ overnight. After washing three times with Tris-buffered saline + Tween 20 , horseradish peroxidase-conjugated secondary antibody was added and incubated at room temperature for 30-60 min. The chemiluminescent substrate (Clarity Western ECL Substrate, Bio-Rad) was added on top of the blot membrane according to the manufacturer's instructions. The signals were captured using a CCD camera-based imager (GE Healthcare).

AAV infectivity assay. AAVs carrying a GFP reporter (AAV-GFP) were used to assess the infection rate of intratumoral AAV delivery. Orthotopic breast tumors were established by transplanting $2 \times 10^{6}$ E0771-dCas9-VP64 cells into the mammary fat pad of 5- to 8-week-old female C57BL/6J mice; 3 and $10 \mathrm{~d}$ after transplantation, $1 \times 10^{11} \mathrm{GCs}$ of AAV-GFP were injected intratumorally into tumorbearing mice. Four days after intratumoral AAV injection, tumor cells, TIICs $\left(\mathrm{CD} 45^{+}\right)$and cells from nontumor organs were harvested. To analyze the persistent expression of AAV-delivered transgene, $12 \mathrm{~d}$ after initial AAV injection (AAV-GFP was injected at day 3 and day 10), tumor cells, TIICs $\left(C D 45^{+}\right)$were harvested. Single tumor cell suspensions were prepared. A LIVE/DEAD Fixable Near-IR Dead Cell Stain Kit was used to distinguish live cells from dead cells. Cells at a density of $10^{7} \mathrm{ml}^{-1}$ were stained with dimethylsulfoxide-dissolved live/dead staining dye and PerCP/Cy5.5-conjugated CD45 antibody in PBS $+2 \%$ FBS, and incubated on ice for $30 \mathrm{~min}$. Stained cells were washed three times before being analyzed and FACS isolated on a BD FACSAria.

Histology and immunohistochemistry. At matched time points, tumors from different treatment group were collected and fixed in $10 \%$ neutral formalin for $2-5 \mathrm{~d}$. Tissues were transferred into $70 \%$ ethanol for long-term storage. Hematoxylin and eosin staining or antibody staining was performed on 3- to $5-\mu \mathrm{m}$ tissue sections using standard procedures at the Yale Pathology Core Facility.
TCR-seq. Mice in different treatment groups were euthanized at 30-40 d posttransplantation. Tumors and spleens were collected and kept in ice-cold $2 \%$ FBS before use. Single-cell suspensions from tumors or spleens were prepared as previously described. The RNA from collected splenocytes and TILs was extracted using TRIzol and an Ambion RNA extraction kit. TCR library prep was performed using the SMARTer Mouse TCR antibody Profiling Kit (Takara) according to the manufacturer's instructions.

TCR-seq data analysis. Raw fastq files from TCR-seq were processed to clonotypes using MiXCR following the author's recommendations ${ }^{59}$. Specifically, the mixcr align function was first used to align to the $\mathrm{V}$ segment transcript, followed by clonotype assembly using mixcr assemble and mixcr exportClones. Samples with fewer than 1,000 UMI counts were removed from further analysis. Subsequently, TCR-seq data were analyzed using the tcR package ${ }^{60}$ to determine clonal proportions and occupied clonal homeostasis in each sample. Various metrics such as Gini-Simpson and Chaol indices were calculated.

\section{Exome sequencing of E0771 and characterization of mutational spectrum.}

E0771 cells and freshly dissected mammary fat pads from healthy, untreated 6- to 8-week-old female C57BL/6J mice were subjected to gDNA extraction following standard protocols. A total of $2 \mu \mathrm{g}$ of gDNA per sample was subjected to exome capture using mouse exome probes (Roche) and then Illumina library preparation following the manufacturer's protocols. Exome-capture, paired-end fastq files were mapped to the mm10 genome using Bowtie v.2.2.9 (ref. ${ }^{61}$ ) and sorted using Samtools ${ }^{62}$. VarScan v.2.3.9 (ref. ${ }^{63}$ ) was then used in somatic mode to call indels and SNPs that were specific to E0771 cells and not found in wild-type C57BL/6J mammary fat-pad tissue. Germline calls were subsequently filtered out. The remaining variants were collapsed to the gene level, and the number of indels or SNPs for each gene was tabulated.

Generation of exome-guided AAV-p-MAEGI. Genes were ranked by the number of somatic variants present in E0771 cells but not in healthy mammary fat pads. Both intronic and exonic variants were included. A set of 1,116 top mutated genes was chosen as differentially mutated genes in E0771. Up to three sgRNAs per gene (three for most of the genes) targeting promoter regions were chosen. A CRISPRa library consisting of 3,839 sgRNAs was designed for the E0771 top mutated gene set (Addgene, no. 129601), pool synthesized (CustomArray) and cloned into the AAV-CRISPRa vector described above. The production of library AAV was done as described above, generating AAV-p-MAEGI for E0771.

\section{Therapeutic testing of AAV-p-MAEGI in mice. Syngeneic TNBC were} established by orthotopic transplantation of $2 \times 10^{6}$ E0771-dCas9-VP64 cells into the mammary fat pad of 5- to 8-week-old female C57BL/6J mice; 5, 12 and $18 \mathrm{~d}$ after tumor induction, 5-10 $\times 10^{10} \mathrm{GCs}$ of AAV-p-MAEGI, AAV-vector or PBS were intratumorally administered to tumor-bearing mice. For testing of the abscopal effect, syngeneic TNBC cases were established by orthotopic transplantation of $2 \times 10^{6}$ E0771 cells into an ipsilateral mammary fat pad, and $2.5 \times 10^{5}$ E0771 cells into the contralateral mammary fat pad; $4,11,18$ and $24 \mathrm{~d}$ after tumor induction, 5-10 $\times 10^{10}$ GCs of AAV-p-MAEGI or AAV-vector were intratumorally administered to the ipsilateral tumor. Tumors were measured every 3-4 d using calipers and their size was calculated using the formula: volume $=\pi / 6 x y z$. Two-way ANOVA was used to compare growth curves between treatment groups. Response to therapy was defined by a method similar to RECIST 1.1 criteria (CR, complete response, where endpoint tumor size $=0$; nCR, near complete response, where tumor size $<50 \mathrm{~mm}^{3}$ for the last two measurements; $\mathrm{PR}$, partial response, where tumor sizes decreased for at least two consecutive measurements; SD, stable disease, where tumor size is between $70 \%$ and $\sim 100 \%$ of initial tumor size at the first treatment; and PD, progressive disease, where tumor size > initial tumor size at the first treatment)

A dual AAV system for in vitro and in vivo delivery of MAEGI. A dual AAVvector system of the CRISPRa (AAV-dCas9, that is, EFs-dSpCas9-spA (Addgene, no. 129598) and AAV-CRISPRa vector, that is U6p-sgSapI-EF1a-MS2-p65-HSF1sPA (Addgene, no. 129602)) was generated by restriction cloning and Gibson assembly. Individual sgRNA or sgRNA libraries were cloned into the AAVCRISPRa plasmid. The dCas9- or U6-sgRNA-EF1a-MS2-p65-HSF1-expressing AAVs were produced using the method described above in Generation of AAVbased MAEGI. For in vitro and in vivo delivery, AAV-dCas9 and AAV-CRISPRa were simultaneously added into cultured cells or co-injected into tumors at a ratio of 1:1.

Therapeutic effects of dual AAV system-delivered p-MAEGI in mice. Syngeneic TNBC cases were established by orthotopic transplantation of $2 \times 10^{6}$ E0771 cells into the mammary fat pad of 5- to 8-week-old female C57BL/6J mice. Then, 4 , 9 (11) and $18 \mathrm{~d}$ after tumor induction, $1-5 \times 10^{10} \mathrm{GCs}$ of AAV-dCas9, together with same titers of AAV-p-MAEGI or AAV-vector, or PBS, were intratumorally administered to tumor-bearing mice. Tumors were measured every 3-4 d using calipers and their size was calculated using the formula: volume $=\pi / 6 x y z$. Two-way ANOVA was used to compare growth curves between treatment groups. 
Statistical analysis. Data analysis was performed using GraphPad Prism v.7 and R 3.5. The unpaired, two-sided, Mann-Whitney test was used to compare two groups unless indicated otherwise. Two-way ANOVA was used to compare multiple groups in the tumor growth curves with two independent variables. $P<0.05$ was considered statistically significant.

Reporting Summary. Further information on research design is available in the Nature Research Reporting Summary linked to this article.

\section{Data availability}

Source data and statistics for non-next-generation sequencing experiments such as tumor studies, flow cytometry, ELISpot and qPCR are provided in an Excel table (see Supplementary Table 1). Processed genomic sequencing data are also provided in an Excel table (see Supplementary Table 2). Original exome sequencing data are available via the National Center for Biotechnology Information Sequence Read Archive (PRJNA553203). Single-cell transcriptome sequencing data are available via the Gene Expression Omnibus (GSE133983). Other data, reagents, methods, computational codes and materials that support the findings of this research are available from the corresponding author upon reasonable request.

\section{Code availability}

Custom codes used to support the findings of this research are available from the corresponding author upon reasonable request.

\section{References}

49. Martin, M. Cutadapt removes adapter sequences from high-throughput sequencing reads. EMBnet.journal 17, 10-12 (2011).

50. Langmead, B., Trapnell, C., Pop, M. \& Salzberg, S. L. Ultrafast and memory-efficient alignment of short DNA sequences to the human genome. Genome Biol. 10, R25 (2009).
51. Joung, J. et al. Genome-scale CRISPR-Cas9 knockout and transcriptional activation screening. Nat. Protoc. 12, 828-863 (2017).

52. Broz, M. L. et al. Dissecting the tumor myeloid compartment reveals rare activating antigen-presenting cells critical for T cell immunity. Cancer Cell 26, 638-652 (2014)

53. Zheng, G. X. et al. Massively parallel digital transcriptional profiling of single cells. Nat. Commun. 8, 14049 (2017).

54. Dobin, A. et al. STAR: ultrafast universal RNA-seq aligner. Bioinformatics 29, 15-21 (2013).

55. Linderman, G. C., Zhao, J. \& Kluger, Y. Zero-preserving imputation of scRNA-seq data using low-rank approximation. bioRxiv https://www.biorxiv. org/content/10.1101/397588v1 (2018).

56. Maaten, Lvd \& Hinton, G. Visualizing data using t-SNE. J. Mach. Learn. Res. 9, 2579-2605 (2008)

57. Maaten, Lvd Accelerating t-SNE using tree-based algorithms. J. Mach. Learn. Res. 15, 3221-3245 (2014).

58. Huang, D. W., Sherman, B. T. \& Lempicki, R. A. Systematic and integrative analysis of large gene lists using DAVID bioinformatics resources. Nat. Protoc. 4, 44-57 (2009).

59. Bolotin, D. A. et al. MiXCR: software for comprehensive adaptive immunity profiling. Nat. Methods 12, 380-381 (2015).

60. Nazarov, V. I. et al. tcR: an R package for $\mathrm{T}$ cell receptor repertoire advanced data analysis. BMC Bioinforma. 16, 175 (2015).

61. Langmead, B. \& Salzberg, S. L. Fast gapped-read alignment with Bowtie 2. Nat. Methods 9, 357-359 (2012).

62. Li, H. et al. The sequence alignment/map format and SAMtools. Bioinformatics 25, 2078-2079 (2009).

63. Koboldt, D. C. et al. VarScan 2: somatic mutation and copy number alteration discovery in cancer by exome sequencing. Genome Res. 22, 568-576 (2012). 


\section{Reporting Summary}

Nature Research wishes to improve the reproducibility of the work that we publish. This form provides structure for consistency and transparency in reporting. For further information on Nature Research policies, see Authors \& Referees and the Editorial Policy Checklist.

\section{Statistics}

For all statistical analyses, confirm that the following items are present in the figure legend, table legend, main text, or Methods section.

$\mathrm{n} / \mathrm{a}$ Confirmed

$\bigotimes$ The exact sample size $(n)$ for each experimental group/condition, given as a discrete number and unit of measurement

$\square$ A statement on whether measurements were taken from distinct samples or whether the same sample was measured repeatedly

The statistical test(s) used AND whether they are one- or two-sided

$\triangle$ Only common tests should be described solely by name; describe more complex techniques in the Methods section.

$\bigotimes$ A description of all covariates tested

$\square$ \ A description of any assumptions or corrections, such as tests of normality and adjustment for multiple comparisons

A full description of the statistical parameters including central tendency (e.g. means) or other basic estimates (e.g. regression coefficient)

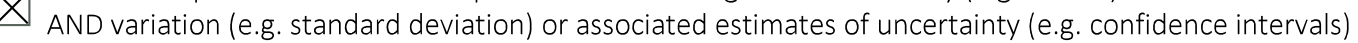

For null hypothesis testing, the test statistic (e.g. $F, t, r$ ) with confidence intervals, effect sizes, degrees of freedom and $P$ value noted Give $P$ values as exact values whenever suitable.

Х $\square$ For Bayesian analysis, information on the choice of priors and Markov chain Monte Carlo settings

Х $\square$ For hierarchical and complex designs, identification of the appropriate level for tests and full reporting of outcomes

$\bigotimes \square$ Estimates of effect sizes (e.g. Cohen's d, Pearson's $r$ ), indicating how they were calculated

Our web collection on statistics for biologists contains articles on many of the points above.

\section{Software and code}

Policy information about availability of computer code

Data collection

Caliper was used for tumor size measurement; BD FACSAria cytometer and Attune NxT Flow Cytometer were used to collect flow cytometry data; Immunospot Reader for ELISpot image and counting.

Data analysis

FlowJo 10.6.0, Aperio ImageScope v12.3.0.5056, Microsoft Excel for Mac 2019, GraphPad Prism 7, R 3.0

Pan-immune cell scRNA-seq data were pre-processed using both established pipelines and custom scripts. Briefly, raw Illumina data files were processed by Cell Ranger 1.3 (10x Genomics), using cellranger mkfastq to wrap Illumina's bcl2fastq to correctly demultiplex sequencing samples and to convert barcode and read data to FASTQ files. Then, cellranger count was used to take FASTQ files and perform alignment to the mouse genome ( $\mathrm{mm} 10$ ), filtering, and UMI counting. Cells with fewer than 2,000 UMIs were subsequently filtered from analysis, leaving a final set of 7,863 cells for further analysis. We next performed imputation using ALRA, which is a conservative strategy for distinguishing technical "dropout" zeros from true biological zeros. After log normalization, we additionally filtered the 27,998 genes/features using a flat cutoff metric such that genes with variance $<0.1$ were excluded, leaving 7,632 genes. Using the final normalized and processed dataset above, we performed t-SNE dimension reduction. Individual data points were colored based on the treatment condition for each cell, or based on unbiased k-means clustering performed on the normalized dataset. Differential expression analyses between clusters and/or treatment conditions was performed by nonparametric two-tailed Mann Whitney test, filtering for genes with variance $>0.1$ across the cell population of interest. Multiple hypothesis correction was performed by the Benjamini-Hochberg method. Significantly differentially expressed genes were defined as having a Benjamini-Hochberg adjusted $p$ $<0.001$. A cutoff of 0 was used for determining whether cells expressed a particular gene of interest. The statistical significance of differences in cell type proportions between AAV-p-MAEGI and AAV-Vector conditions was assessed by two-tailed Fisher's exact test. Gene ontology analysis was performed in DAVID.

Raw fastq files from TCR-seq were processed to clonotypes using MiXCR 2.1.5 following the author recommendations. Specifically, the mixcr align function was first used to align to the $V$ segment transcript, followed by clonotype assembly using mixcr assemble and mixcr exportClones. Samples with fewer than 1,000 UMIs were removed from further analysis. Subsequently, TCR-seq data were analyzed using the tcR package to determine clonal proportions and occupied clonal homeostasis in each sample. Various metrics such as the GiniSimpson and Chao1 indices were calculated.

Exome capture paired-end fastq files were mapped to the mm10 genome using Bowtie v2.2.9 67, and sorted by Samtools. VarScan v2.3.9 
69 was then used in somatic mode to call indels and SNPs that were specific to E0771 cells and not found in wildtype C57BL/6J mammary fat pad tissue. Germline calls were subsequently filtered out. The remaining variants were collapsed to the gene level, and the number of indels or SNPs for each gene was tabulated.

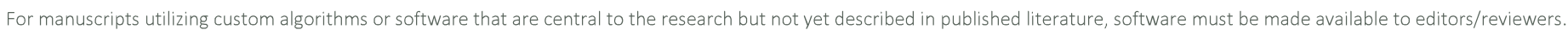
We strongly encourage code deposition in a community repository (e.g. GitHub). See the Nature Research guidelines for submitting code \& software for further information.

Data

Policy information about availability of data

All manuscripts must include a data availability statement. This statement should provide the following information, where applicable:

- Accession codes, unique identifiers, or web links for publicly available datasets

- A list of figures that have associated raw data

- A description of any restrictions on data availability

Accessions

Exome sequencing data are available via NCBI SRA (PRJNA553203). Single cell transcriptome sequencing data are available via GEO (GSE133983).

Data and resource availability

Source data for non-NGS experiments such as tumor studies, flow cytometry, QPCR, ELISPOT, and respective statistics are provided in the supplementary tables. Genome sequencing and gene expression data are deposited to SRA and GEO with accession numbers as above, with processed data provided in supplementary tables. Other data, reagents, methods, and materials that support the findings of this research are available to the academic community from the corresponding author upon reasonable request.

\section{Field-specific reporting}

Please select the one below that is the best fit for your research. If you are not sure, read the appropriate sections before making your selection. Х Life sciences Behavioural \& social sciences Ecological, evolutionary \& environmental sciences

For a reference copy of the document with all sections, see nature.com/documents/nr-reporting-summary-flat.pdf

\section{Life sciences study design}

All studies must disclose on these points even when the disclosure is negative.

Sample size

Sample size was empirically determined for a statistical significant statement. Prior estimation of number of mice used were estimated based on similar tumor models in the field. No statistical methods were used to predetermine sample sizes. See Methods and figure legends for details.

Data exclusions No data were excluded.

Replication All animal and in vitro experiment has multiple replicates. Key experiments were repeated 2-4 times. All the experiments were reproducible with concordant results. See Methods and figure legends for details.

Randomization The tumor-bearing mice were randomized by littermates and cages housed into different groups so that all the groups had similar initial tumor volume. See Methods and figure legends for details.

Blinding Investigators were not blinding for the acquisition and analysis of data. The conclusions in the manuscript were based on multiple independent experiments and technical replicates as well as statistical significance.

\section{Reporting for specific materials, systems and methods}

We require information from authors about some types of materials, experimental systems and methods used in many studies. Here, indicate whether each material, system or method listed is relevant to your study. If you are not sure if a list item applies to your research, read the appropriate section before selecting a response.

\begin{tabular}{|c|c|}
\hline $\mathrm{n} / \mathrm{a}$ & Involved in the study \\
\hline - & X Antibodies \\
\hline \begin{tabular}{|r} 
\\
\end{tabular} & $\bigotimes$ Eukaryotic cell lines \\
\hline Х & $\square$ Palaeontology \\
\hline L & $\bigotimes$ Animals and other organisms \\
\hline 邓 & $\square$ Human research participants \\
\hline Х & $\square$ Clinical data \\
\hline
\end{tabular}

\begin{tabular}{l|l}
\multicolumn{2}{l}{ Methods } \\
\hline n/a & Involved in the study \\
$\square$ & $\square$ ChIP-seq \\
$\square$ & $\square$ Flow cytometry \\
$\square$ & $\square$ MRI-based neuroimaging
\end{tabular}


Antibodies used

Validation
All antibodies used were detailed described with name, vendor, catalog number, clone number.

Antibodies used for in vivo experoments: anti-CD4 (BioXcell BE0003-1), anti-CD8 (BioXcell BE0117), anti-CTLA4 (BioXcell,BE0164). Antibodies used for western blot: S. aureus CRISPR/Cas9 monoclonal antibody (Diagenode C15200230-10), anti-Gapdh:(SantaCruz sc-32233)

Antibodies used for flow cytometry: APC/Cy7 anti-mouse CD45.2 (Biolegend 109823), PE anti-mouse CD3 (Biolegend 100308), APC anti-mouse CD8a (Biolegend 100712), FITC anti-mouse CD4 (Biolegend 100406), APC anti-mouse CD45.2 (Biolegend 109814), APC/Cy7 anti-mouse IA/IE (Biolegend 107268), EP/Cy7 anti-mouse CD4 (Biolegend 100422), APC/Cy7 anti-mouse CD8a (Biolegend 100714), PerCP/Cy5.5 anti-mouse CD45 (Biolegend 103132), PerCP/Cy5.5 anti-mouse IA/IE (Biolegend 107626), FITC anti-mouse CD3 (Biolegend 100306), FITC anti-mouse/human CD11b (Biolegend 101205), PE anti-mouse F4/80 (Biolegend 123109), PE/Cy5 anti-mouse CD24 (Biolegend 101811), PE/Dazzle 594 anti-mouse CD11c (Biolegend 117348), APC anti-mouse Ly6C (Biolegend 128016), anti-mouse CD16/32 (Biolegend 101302), APC anti-mouse H-2Kb-SIINFEKL (Biolegend 141606), PE antimouse H-2Kb-SIINFEKL (Biolegend 141604). See supplementary tables for details.

All the primary antibodies used in flow cytometry, in vivo experiments and western blot were validated by manufacturers, and data are available on the manufacturer's website. All used antibodies are commercially available.

\section{Eukaryotic cell lines}

Policy information about cell lines

Cell line source(s)

Authentication

Mycoplasma contamination

Commonly misidentified lines (See $\underline{I C L A C}$ register)
E0771 was from CH3. Pan02 and B16F10 were from ATCC. HEK293FT was from ThermoFisher.

All cell lines used have been authenticated by the original vendors. Cell lines from ATCC have been thoroughly tested and authenticated, using morphology, karyotyping, and PCR based approaches to confirm the identity of cell lines and to rule out both intra- and interspecies contamination.

All cell lines used in this study were tested negative for mycoplasma contamination.

No commonly misidentified cell lines were used.

\section{Animals and other organisms}

Policy information about studies involving animals; ARRIVE guidelines recommended for reporting animal research

Laboratory animals

Wild animals

Field-collected samples

Ethics oversight
Various animals were used in this study. 5-10 week with a median age of 7 week old C57BL/6J mice, 7 week old Nude (Foxn1-/-) mice, or 6-8 week old with a median age of 7 week old Rag1-/- mice were used for experiments. Female mice were used for breast cancer models. Male mice were used for melanoma and PDAC models. See method and figure legend sections for details.

This study does not invovle wild animals.

This study did not involve samples collected from the field.

All animal work was performed under the guidelines of Yale University Institutional Animal Care and Use Committee (IACUC) with approved protocols (Chen-2015-20068, Chen-2018-20068), and was consistent with the Guide for Care and Use of Laboratory Animals, National Research Council, 1996 (Institutional Animal Welfare Assurance No. A-3125-01). All recombinant DNA work was performed under the guidelines of Yale University Environmental Health and Safety (EHS) committee with an approved protocol (Chen-rDNA-15-45).

Note that full information on the approval of the study protocol must also be provided in the manuscript.

\section{Flow Cytometry}

\section{Plots}

Confirm that:

\The axis labels state the marker and fluorochrome used (e.g. CD4-FITC).

\The axis scales are clearly visible. Include numbers along axes only for bottom left plot of group (a 'group' is an analysis of identical markers).

$\bigotimes$ All plots are contour plots with outliers or pseudocolor plots.

\A numerical value for number of cells or percentage (with statistics) is provided.

\section{Methodology}

Sample preparation

Samples were prepared as described in the methods section. 


\section{Software}

FlowJo 10.6.0 (Treestar) was used for data analysis.

Cell population abundance

Gating strategy

N/A

W Tick this box to confirm that a figure exemplifying the gating strategy is provided in the Supplementary Information. 